\title{
Equilibrium in Securities Markets with Heterogeneous Investors and Unspanned Income Risk ${ }^{\mathrm{a}}$
}

May 2, 2011

\author{
Peter Ove Christensen \\ School of Economics and Management, \\ Aarhus University, \\ DK-8000 Aarhus C, Denmark \\ email: pochristensen@econ.au.dk \\ Kasper Larsen \\ Department of Mathematical Sciences, \\ Carnegie Mellon University, \\ Pittsburgh, PA 15213 \\ email: kasperl@andrew.cmu.edu
}

\begin{abstract}
Claus Munk ${ }^{b}$
School of Economics and Management \& Dept. of Mathematical Sciences,

Aarhus University,

DK-8000 Aarhus C, Denmark

email: cmunk@econ.au.dk
\end{abstract}

\footnotetext{
${ }^{\text {a }}$ We would like to thank the participants in the European Winter Finance Summit 2010 and seminars at University of Copenhagen, Goethe University Frankfurt and, in particular, Costis Skiadas for many helpful comments and suggestions. The comments by the editor and two anonymous referees are gratefully acknowledged and significantly helped improve the paper. All remaining errors are our own. All three authors acknowledge financial support from the Danish Center for Accounting and Finance (D-CAF).

${ }^{\mathrm{b}}$ Corresponding author. Full address: School of Economics and Management, Aarhus University, Bartholin's Alle 10, Building 1322, DK-8000 Aarhus C, Denmark.
} 


\title{
Equilibrium in Securities Markets with Heterogeneous Investors and Unspanned Income Risk
}

\begin{abstract}
In a finite time horizon, incomplete market, continuous-time setting with dividends and investor incomes governed by arithmetic Brownian motions, we derive closed-form solutions for the equilibrium risk-free rate and stock price for an economy with a finite set of heterogeneous CARA investors and unspanned income risk. In equilibrium, the Sharpe ratio is the same as in an otherwise identical complete market economy, whereas the riskfree rate is lower and, consequently, the stock price is higher. The reduction in the risk-free rate is highest when the more risk-averse investors face the largest unspanned income risk. In numerical examples with reasonable parameters, the risk-free rate is reduced by several percentage points.
\end{abstract}

Keywords. Unspanned income, heterogeneous preferences, continuous-time equilibrium, risk-free rate puzzle, equity premium, incomplete markets, Brownian motion

JEL classification. G12, G11, D53 


\section{Introduction}

Labor income is an important source of wealth for most individuals (Heaton and Lucas 2000; Campbell 2006) with a potentially large impact on their consumption and portfolio decisions and, consequently, on the equilibrium securities prices. However, because labor income typically has a large unhedgeable component, solving for the optimal decision rules and equilibrium prices is very difficult. Closed-form solutions for the optimal individual decisions with unspanned income have been derived in a few settings with either preferences for consumption over an infinite time interval or for consumption at a single future date. We derive closed-form solutions for the more appropriate case of consumption over a finite time interval for an incomplete market, continuous-time setting with negative exponential (CARA) utilities and dividends and labor income governed by arithmetic Brownian motions. More importantly, using these individually optimal decision rules, we derive the first closed-form solution in the literature for the equilibrium risk-free rate and the equilibrium stock price in a setting with a finite set of heterogeneous investors as well as unspanned income risk both at the individual and the aggregate level.

For fixed aggregate consumption dynamics, the equilibrium stock market Sharpe ratio is the same as in an otherwise identical dynamically complete market economy in which all risks are spanned, whereas the equilibrium risk-free rate (and the expected stock return) is lower and, consequently, the equilibrium stock price is higher. Our closed-form equilibrium thus explains the findings of earlier numerical studies (references are given below) that persistent non-hedgeable income shocks can help in explaining the risk-free rate puzzle. ${ }^{1}$ Our main contribution to the literature is the closed-form equilibrium for the incomplete market, continuous-time economy. This allows us to quantify and to clearly explain the underlying economics of these earlier numerical findings.

The lower risk-free rate in our equilibrium is due to the fact that, in equilibrium, the investors evaluate their future unspanned income risk using their personal risk tolerance as opposed to the aggregate risk tolerance in the economy which they are using for evaluating risks that can be efficiently shared in the market. Hence, the risk premium for aggregate consumption risk in the determination of the equilibrium risk-free rate is higher in the setting with unspanned income risks than in settings in which these risks can be efficiently shared. Moreover, our equilibrium demonstrates the importance of the distribution of income risk across individuals: the reduction in the risk-free rate is highest when the more risk-averse investors face the largest unspanned income risk.

\footnotetext{
${ }^{1}$ The risk-free rate puzzle, first identified by Weil (1989), refers to the observation that the historical riskfree rate is smaller than the risk-free rate predicted by simple consumption-based representative agent models. In order to match the observed equity premium in these models, the risk aversion parameter has to be very high, but then the risk-free rate becomes too high, unless we also allow for a negative time preference rate.
} 
Our economy is formulated in a continuous-time, finite time horizon model with a single consumption good, a risk-free asset and a single risky asset. The risky asset is a claim to an exogenous dividend stream represented by an arithmetic Brownian motion. There are a finite number of consumer-investors maximizing time-additive negative exponential utility of consumption, with heterogeneity in their subjective time preference rates and in their absolute risk aversions. Each investor receives an exogenous income stream represented by another arithmetic Brownian motion, which is imperfectly correlated with the stock's dividend process and, therefore, also imperfectly correlated with the stock's price process. In other words, each investor's income process contains an unhedgeable risk component.

Our analysis is performed by first conjecturing the form of the equilibrium risk-free rate and of the equilibrium stock price, and then we solve for each investor's optimal consumption and investment strategy. Secondly, by aggregating over investors we find an equilibrium consistent with our conjecture. In particular, the equilibrium risk-free rate, stock price volatility, and Sharpe ratio are all deterministic processes.

First, we solve the utility maximization problem of each investor assuming a deterministic risk-free rate, stock price volatility, and Sharpe ratio. This result makes a contribution to the literature on optimal consumption and portfolio choice. The introduction of spanned income risk does not complicate the solution of multi-period utility maximization problems (Merton 1971; Bodie, Merton, and Samuelson 1992). However, when the income process is partially unspanned, the investor's optimization problem is much more complicated. Explicit solutions have been found in special cases involving CARA utility, a normally distributed income stream, a constant risk-free rate, and a constant drift and volatility of the stock price. ${ }^{2}$ Svensson and Werner (1993) and Wang (2006) consider infinite time horizon settings where a transversality condition has to be imposed on the utility maximization problem. ${ }^{3}$ The transversality condition restricts the rate at which the debt of the investor can grow, but does not force the investor to ever pay back the debt and can thus lead to excessive borrowing compared to the finite horizon setting. In contrast, in our finite horizon model, we ensure that the debt of the investor equals zero at the end of the horizon. ${ }^{4}$ Henderson (2005) assumes a finite time horizon, as we do, but considers utility of terminal wealth

\footnotetext{
${ }^{2}$ Several recent papers have numerically solved for optimal consumption and portfolio strategies in more general settings, e.g., Cocco, Gomes, and Maenhout (2005), Koijen, Nijman, and Werker (2010), Lynch and Tan (2011), Munk and Sørensen (2010), and Viceira (2001).

${ }^{3}$ The models of Svensson and Werner (1993) and Wang (2006) differ slightly with respect to the specification of the income process. Furthermore, in the model of Wang (2006) only a risk-free asset is traded, whereas Svensson and Werner (1993) allow for risky assets. In similar settings, Wang (2004, 2009) investigates the impact of unobservable or partially observable income growth on consumption and investment decisions.

${ }^{4}$ The investor can borrow against future income, but admissible strategies are defined so that terminal financial wealth is non-negative to ensure that the investor does not end up indebted. As a consequence, the proof of the optimal strategies involves a mathematically interesting Ornstein-Uhlenbeck type bridge process.
} 
only. ${ }^{5}$ Henderson conjectures [last paragraph of her concluding remarks] that "if we were to incorporate consumption, it is unlikely that the model could be solved analytically." Our results do indeed generalize Henderson's findings to the case of consumption over a finite time horizon.

We show that the optimal consumption at any date is equal to what the investor could get by spreading out evenly - annuitizing - the investor's perceived total wealth over the remaining consumption period, in the spirit of the permanent-income hypothesis of Friedman (1957). The perceived total wealth is the sum of the financial wealth, corrected upwards due to the future investment opportunities, and an appropriate measure of human wealth. Any persistent idiosyncratic income shock, positive or negative, leads to an immediate identical change in consumption. The optimal stock investment is a combination of the standard myopic term and an income hedge term which basically "undoes" the stock-like risk inherent in the income process. Due to precautionary savings, the optimal risk-free investment is increasing in the magnitude of the unspanned income risk component.

Next, we aggregate over the individual consumer-investors and impose the market clearing conditions. We derive simple explicit solutions (in terms of the exogenously specified primitives of the economy) for the equilibrium stock price and risk-free rate confirming the conjecture we used in the individual utility maximization problems, i.e., the risk-free rate, the Sharpe ratio, and the volatility of the stock are all deterministic processes. We show that an increase in the fraction of income risk which is spanned by the stock market implies a higher stock market Sharpe ratio and a higher risk-free rate.

We compare our equilibrium to the equilibrium in an otherwise identical economy with all income risk being spanned, but the same heterogeneous preferences and the same aggregate consumption dynamics. In such an economy, the determination of the equilibrium risk-free rate and stock market Sharpe ratio is immediate. In both economies, the Sharpe ratio is the product of the volatility of aggregate consumption and the reciprocal of the aggregate absolute risk tolerance (the latter being lower relative to an economy with homogeneous risk aversions). In both economies, the equilibrium risk-free rate is the sum of three components: (1) a risktolerance weighted average of the investors' time preference rates, where the weighted average may be lower or higher than the (non-weighted) average time preference rate; ${ }^{6}$ (2) the product of the expected growth rate of aggregate consumption and the reciprocal of the aggregate risk tolerance; and (3) a negative term due to precautionary savings. Neither the first nor the second term are affected by the presence of unspanned income risk. The third term is

\footnotetext{
${ }^{5}$ Henderson (2005) also finds near-explicit solutions for more general income processes. Duffie and Jackson (1990) and Teplá (2000) derive similar solutions for investors receiving an unspanned income only at the terminal date.

${ }^{6}$ This phenomenon is also discussed by Gollier and Zeckhauser (2005), who consider an economy with heterogenous investors but no uncertainty.
} 
the channel through which unspanned income risk affects the equilibrium risk-free rate. The demand for precautionary savings is higher when income risk cannot be hedged, which in turn produces a lower equilibrium risk-free rate. Other things being equal, the reduction in the risk-free rate is higher, the larger the unspanned component of the income shocks is. The heterogeneity in risk aversion is crucial for the magnitude of the reduction in the risk-free rate. The reduction is highest when the more risk-averse investors face the largest unspanned income risk. In numerical examples with reasonable parameters, we find a reduction in the risk-free rate of several percentage points. Since the stock market Sharpe ratio is the same, the equilibrium expected return on the stock is also lower than in the economy with identical aggregate consumption dynamics but fully spanned income risk.

Of course, it is straightforward to include labor income in a representative agent modeling framework (see, e.g., Santos and Veronesi 2006), but the representative agent framework requires complete or effectively complete markets, which is not possible when investors have non-traded income streams. Various papers search numerically for equilibrium asset prices in models with a few investors facing idiosyncratic income shocks. For example, Telmer (1993) studies a model with two investors having identical preferences and initial endowments, but later their incomes may diverge due to transitory idiosyncratic shocks. The investors can only trade in a risk-free asset and are thus unable to hedge the income shocks. The investors self-insure against adverse income shocks by buffer savings and, therefore, the equilibrium interest rate is lower, but for reasonable parameterizations the reduction is small. Similar computational results are reported by Aiyagari and Gertler (1991) and Heaton and Lucas (1996), whereas Levine and Zame (2002) arrive at a similar conclusion in a theoretical study. Shocks to individual incomes must be non-hedgeable, persistent, and heterogeneous across individuals to have a significant impact on equilibrium asset prices, cf. Constantinides (2002).

Constantinides and Duffie (1996) assume the investors have identical constant relative risk aversion (CRRA) preferences but are subject to persistent non-hedgeable shocks. Given virtually any pattern of stock and bond prices, individual income processes can be derived so that the (no-trade) equilibrium in their model is consistent with these prices. Hence, such labor income shocks can potentially explain the standard asset pricing puzzles. In particular, if the cross-sectional dispersion is counter-cyclical and sufficiently large, the model prices are consistent with the observed high equity premium. Cochrane (2005, Ch. 21) argues that cross-sectional income data do not show such large dispersion but agrees that persistent idiosyncratic income shocks may have a substantial impact on equilibrium asset prices. In contrast to Constantinides and Duffie (1996), we allow for heterogeneous preferences and derive the equilibrium (with trading) from assumptions on the primitives.

Some papers derive equilibria in Bewley-type models with idiosyncratic income risk but no non-hedgeable aggregate risk, cf. Bewley (1986). In these models, the traditional market 
clearing conditions are, by reference to a law of large numbers, substituted with market clearing conditions stated in terms of expected per capita demand and supply such that in the "aggregate," there is no non-hedgeable risk. ${ }^{7}$ For example, Wang (2003) considers a model with only a risk-free asset and individuals having homogeneous CARA preferences and independent and identically distributed incomes. By assuming that there are infinitely many individuals and that (by the law of large numbers) the cross-sectional distribution of realized income is equal to the stationary distribution of each individual's income, "aggregate" income - which equals "aggregate" consumption - is constant over time. The savings of each individual is thus determined by the difference between the constant return on savings (the risk-free rate) and the consumption impatience (the subjective time preference rate), as well as the precautionary savings induced by the individual income risk. ${ }^{8}$ By investor homogeneity, the equilibrium savings of each individual must be zero. Consequently, the equilibrium riskfree rate equals the subjective time preference rate minus a precautionary savings term.

Similarly, in a discrete-time Markov-chain setting, Krueger and Lustig (2010) provide conditions under which non-hedgeable idiosyncratic income risk will lower the risk-free rate, but not affect the market prices of risk. The conditions include having a continuum of agents with identical CRRA preferences, idiosyncratic income risk which is independent of aggregate risk, and solvency constraints which are proportional to aggregate income. Moreover, aggregate income must be a constant fraction of aggregate consumption and is thus spanned by a complete set of aggregate consumption claims, while the assumptions of a continuum of identical CRRA investors ensure that the realizations of idiosyncratic income shocks have no impact on "aggregate" demand and supply (and, thus, no impact on equilibrium securities prices). Qualitatively, this model produces similar results as our model; however, it relies on a continuum of identical investors, and it does not allow for non-hedgeable aggregate income risk. $^{9}$

Our model has finitely many individuals with heterogenous preferences and income risks, and aggregate income is risky and non-hedgeable (i.e., each idiosyncratic income shock is

\footnotetext{
7 "The clue is to change the definition of feasibility: the balance between supply and demand is required to hold only in expected value across all states and no longer for each single state." Malinvaud (1972, pp. 313).

${ }^{8}$ In addition, each investor has savings due to changes in expected future income, but again the homogeneity of investors and the law of large numbers imply that the "aggregate" of these savings is equal to zero.

${ }^{9}$ The application of the law of large numbers in Bewley-type models is non-trivial. For example, if the individual demand for a risk-free asset (in zero net-supply) is affected by identically distributed idiosyncratic income shocks, the law of large numbers implies that the realized aggregate net-demand per capita is equal to the expected net-demand per capita in the limit with an infinite number (or a continuum) of investors. However, the aggregate realized net-demand is not equal to zero. Hence, there is a need for a mechanism (for example, an infinitely large risk neutral insurance system) to absorb or distribute the individually negligible but large aggregate realized deviations in the traditional market clearing condition that aggregate net-demand must be equal to zero; see, for example, Malinvaud (1972, 1973), Judd (1985), and Uhlig (1996) for further discussion of conceptual and technical issues in these models.
} 
by construction a shock to aggregate consumption, cf. Den Haan 2001). Instead of making reference to a law of large numbers and a market clearing condition stated in terms of expected demand and supply, we ensure a deterministic risk-free rate and market price of risk (in order to be able to solve the individual investors' consumption-portfolio problems in closed-form) by imposing strong conditions on the stochastic processes for aggregate income and dividends and by assuming CARA preferences in order to eliminate wealth effects. In particular, CARA preferences and independent increments for aggregate income and dividends (as for arithmetic Brownian motions) imply deterministic equilibrium risk-free rates and market prices of risk. Calvet (2001) considers a discrete-time economy with a finite number of identical CARA investors and normally distributed non-hedgeable income, and derives a closed-form recursion for the equilibrium risk-free rate which shows that the risk-free rate is lower than in an otherwise identical complete market economy due to a precautionary demand for savings. However, Calvet assumes that aggregate income is deterministic such that the individual income risks only pertain to how the deterministic aggregate income is allocated among the investors. Because the investors have identical CARA utilities (without wealth effects), the realized cross-sectional distribution of income has no impact on equilibrium securities prices (which then become deterministic). Other papers exploring the impact of income risk on asset prices include Huggett (1993), Lucas (1994), Krusell and Smith (1997), Storesletten, Telmer, and Yaron (2007), and Krusell, Mukoyama, and Smith (2010).

Our model highlights the role of persistent non-hedgeable income shocks and the crosssectional distribution of those shocks in explaining the risk-free rate puzzle. A few papers have offered alternative explanations of the risk-free rate puzzle in rational asset pricing models. In a calibrated version of a relatively simple overlapping generations model, Constantinides, Donaldson, and Mehra (2002) present numerical results showing that borrowing constraints restraining young individuals from borrowing against future labor income can significantly reduce the risk-free rate and partly explain the risk-free rate puzzle, but they do not discuss the role of unspanned income risk in the determination of the risk-free rate. Bansal and Yaron (2004) consider an economy with long-run consumption risk and a representative agent displaying Epstein-Zin recursive utility. The ability to disentangle the risk aversion from the elasticity of intertemporal substitution adds important flexibility in matching the low historical risk-free rate. Bansal and Yaron (2004) do not include labor income explicitly and by virtue of their representative agent framework, there is no room for unspanned income risk.

Finally, we briefly explore the link between our equilibrium with unspanned income and an equilibrium where individuals have heterogeneous expectations. ${ }^{10}$ We show that our equilibrium can also be interpreted as an equilibrium in an economy where agents receive no

\footnotetext{
${ }^{10}$ We are grateful to Costis Skiadas for suggesting this link.
} 
income, but have certain heterogeneous expectations and certain time-dependent subjective time preference rates. This relates our paper to the recent literature on the impact of heterogeneous expectations on asset prices. For example, David (2008) investigates a model with agents having heterogeneous beliefs about the growth rate of dividends and total output in the economy, which affects their asset demands as they engage in speculative bets on the relative accuracy of their predictions. The speculative trading changes the equilibrium risk-free rate and equity premium. A calibrated version of the model can partially explain the equity premium puzzle and the risk-free rate puzzle, but only when agents have constant relative risk aversions smaller than one. Xiong and Yan (2010) derive equilibrium bond prices in a model where agents have different beliefs about the long-run inflation target and they show that this heterogeneity helps in explaining the observed high volatility of bond yields and the failure of the expectation hypothesis. Other recent papers exploring the impact of heterogenous beliefs on asset prices include Başak (2000), Dumas, Kurshev, and Uppal (2009), Gallmeyer and Hollifield (2008), and Jouini and Napp (2009).

The paper proceeds as follows. Section 2 presents the economy, i.e., the individuals' preferences and endowments as well as the tradeable assets. Section 3 solves for any given investor's optimal consumption and investment strategy under the assumption that the riskfree rate, the excess expected return and the volatility of the stock are deterministic functions of time. Section 4 then shows by aggregation over investors that the conjectured form of the equilibrium is correct and pins down the precise equilibrium risk-free rate and stock price

dynamics. Section 5 provides the link to heterogenous expectations models. Finally, we conclude in Section 6. The appendix contains the proof of our Theorem 1.

\section{The economy}

We consider a continuous-time economy over the time interval $[0, T], T \in(0, \infty)$. The economy offers a single consumption good, which is the numéraire. Two financial assets are available for trading throughout the time interval. The first asset is a risk-free asset with $B_{t}$ denoting its time $t$ price initialized at $B_{0}=1$. The second asset is a risky asset, which is a claim to a continuous exogenous dividend process $D=\left(D_{t}\right)$ evolving as

$$
d D_{t}=\mu_{D}(t) d t+\sigma_{D}(t) d W_{t}, \quad t \in[0, T], \quad D_{0} \in \mathbb{R},
$$

for two deterministic functions of time $\mu_{D}$ and $\sigma_{D}$. In (1), the process $W=\left(W_{t}\right)$ is a standard Brownian motion, i.e., the dividend process $D$ is an arithmetic Brownian motion. We assume that the risky asset is in unit net supply and denote its time $t$ price by $S_{t}$. We later specify the individuals such that, in equilibrium, the risk-free asset provides a deterministic rate of 
return, i.e.,

$$
d B_{t}=r(t) B_{t} d t, \quad t \in[0, T]
$$

where $r$ is a deterministic function of time. The equilibrium price dynamics of the risky asset will have the form

$$
d S_{t}=\left(S_{t} r(t)+\mu_{S}(t)-D_{t}\right) d t+\sigma_{S}(t) d W_{t}, \quad t \in[0, T], \quad S(T)=0,
$$

for some deterministic functions of time $\mu_{S}$ and $\sigma_{S}$. Here, $\mu_{S}$ denotes the total expected excess return over the risk-free rate and $\sigma_{S}$ is the (absolute) price volatility. The ratio $\lambda_{S}(t)=\mu_{S}(t) / \sigma_{S}(t)$ is the market price of risk associated with the Brownian motion $W$ and is also identical to the Sharpe ratio of the risky asset $S$. For later use, we introduce the notation

$$
\beta(t, s)=\exp \left(-\int_{t}^{s} r(u) d u\right), \quad 0 \leq t \leq s \leq T,
$$

which denotes the time $t$ price of a zero-coupon bond with a unit payment at time $s$. Furthermore, the process

$$
A(t)=\int_{t}^{T} \beta(t, u) d u, \quad t \in[0, T]
$$

denotes the annuity factor for $[t, T]$, i.e., the time $t$ value of a continuous payment stream with a unit payment per period until the terminal date $T$. Note that $A(t) \rightarrow 0$ as $t \rightarrow T$ and, therefore, $A(t)^{-1} \rightarrow \infty$ for $t \rightarrow T$.

The economy is populated by $I$ consumer-investors all living on the finite time interval $[0, T]$ and all having time-additive negative exponential utility of consumption. The investors may have different degrees of risk aversion as well as different time preference rates. Investor $i$ is thus maximizing $\mathbb{E}\left[\int_{0}^{T} U_{i}\left(t, c_{t}\right) d s\right]$, where

$$
U_{i}(t, c)=-e^{-\delta_{i} t} e^{-a_{i} c}, \quad c \in \mathbb{R}, \quad t \in[0, T],
$$

and $\delta_{i} \geq 0$ and $a_{i} \geq 0$ are the subjective time preference rate and the absolute risk aversion coefficient of investor $i$, respectively. Each investor $i$ receives income according to an exogenous income rate process $Y_{i}=\left(Y_{i t}\right)$, i.e., the cumulative income up to time $t \in[0, T]$ is given by $\int_{0}^{t} Y_{i u} d u$. We assume that $Y_{i}$ has the dynamics

$$
d Y_{i t}=\mu_{Y i}(t) d t+\sigma_{Y i}(t)\left(\rho_{i} d W_{t}+\sqrt{1-\rho_{i}^{2}} d Z_{i t}\right), \quad t \in[0, T]
$$

where $\mu_{Y i}$ and $\sigma_{Y i}$ are deterministic functions of time, and where $Z_{i}=\left(Z_{i t}\right)$ is a standard 
Brownian motion so that $W, Z_{1}, \ldots, Z_{I}$ are independent. ${ }^{11}$ The constant $\rho_{i} \in[-1,+1]$ controls the income-stock correlation. Hence, our model allows for both common income risk which is hedgeable via trading in the risky asset and for idiosyncratic unspanned income risk that cannot be hedged. Note that all shocks to the income rate are persistent.

We conclude this section by making two assumptions on the market structure. The first assumption is made on the primitives of the economy and is therefore completely exogenous.

Assumption 1. The deterministic functions $\left(\sigma_{Y i}, \sigma_{D}, \mu_{Y i}, \mu_{D}\right), i=1, \ldots, I$, are continuous and finitely valued on the interval $[0, T]$.

The second assumption is made on the price dynamics and, thus, has to be verified by the equilibrium parameter processes.

Assumption 2. The interest rate $r$ is a deterministic and continuous function on $[0, T]$ which satisfies

$$
\lim _{s \uparrow T} \int_{0}^{s} A(t)^{-1} d t=+\infty .
$$

The deterministic functions $\left(\mu_{S}, \sigma_{S}\right)$ are continuous on the interval $[0, T]$ and are such that the market price of risk $\lambda_{S}(t)=\mu_{S}(t) / \sigma_{S}(t)$ is well-defined and belongs to $\mathcal{L}^{p}$ for all $p \geq 1$, i.e.,

$$
\int_{0}^{T}\left(\lambda_{S}(t)\right)^{p} d t<\infty
$$

As we shall see, Assumption 1 implies that the equilibrium parameters $r, \mu_{S}$, and $\sigma_{S}$ automatically satisfy Assumption 2. Indeed, Assumption 1 leads to a bounded deterministic interest rate and such rates satisfy the integrability requirement: if $r(t) \geq \underline{r}$ for all $t \in[0, T]$ for some constant $\underline{r}$, we have

$$
\beta(t, s)=\exp \left(-\int_{t}^{s} r(u) d u\right) \leq e^{-\underline{r}(s-t)}, \quad 0 \leq t \leq s \leq T
$$

\footnotetext{
${ }^{11}$ The analysis goes through when $Z_{1}, \ldots, Z_{I}$ are allowed to be correlated, but at the expense of increased notational complexity. Similarly, it is not crucial for our results that the individual income processes are arithmetic Brownian motions, but rather that the aggregate income process has independent increments in order to ensure a deterministic equilibrium risk-free rate and market price of risk. We could, for example, allow for mean reversion in the individual income processes and still solve the individual investors' problems and get closed-form solutions for the deterministic equilibrium risk-free rate and market price of risk, as long as the aggregate income process has no mean reversion. Moreover, it is not crucial that the uncertainty is driven by Brownian motions. Certain types of jump processes have also independent increments (and, consequently, deterministic posterior volatilities), and the equilibrium can be solved in closed-form in such settings as well. On the other hand, without independent increments in the aggregate income process, the equilibrium risk-free rate and market price of risk become stochastic, and it seems impossible to solve in closed-form the individual investors' problems in such settings.
} 
Consequently,

$$
A(t) \leq \frac{1}{\underline{r}}\left(1-e^{-\underline{r}(T-t)}\right),
$$

and the reciprocal of the right-hand-side does not have a finite integral over $[0, T]$.

\section{The individual investor's problem}

In this section, we solve the utility maximization problem of each investor. In order to simplify the notation, we suppress the $i$ subscripts identifying the investor throughout this section.

\subsection{Admissible strategies}

The investor has to choose a consumption process $c=\left(c_{t}\right)$ and an investment process $\theta=\left(\theta_{t}\right)$, where $\theta_{t}$ represents the number of units of the risky asset owned by the investor at time $t$ for $t \in[0, T]$. The remaining wealth is invested in the risk-free asset. Given a consumption and investment strategy $(c, \theta)$, we let $X_{t}^{(c, \theta)}$ denote the investor's financial wealth at time $t$ and let $x \in \mathbb{R}$ be the investor's initial wealth. For $t \in[0, T]$, the wealth dynamics is given by

$$
\begin{aligned}
d X_{t}^{(c, \theta)} & =\left(X_{t}^{(c, \theta)}-\theta_{t} S_{t}\right) r(t) d t+\theta_{t}\left(d S_{t}+D_{t} d t\right)+\left(Y_{t}-c_{t}\right) d t \\
& =\left(X_{t}^{(c, \theta)} r(t)+Y_{t}-c_{t}\right) d t+\theta_{t} \mu_{S}(t) d t+\theta_{t} \sigma_{S}(t) d W_{t}
\end{aligned}
$$

with $X_{0}^{(c, \theta)}=x$. Note that the dividend process $D$ does not appear in the wealth dynamics. In order to ensure that $X^{(c, \theta)}$ is well-defined, we require that $c \in \mathcal{L}^{1}$ and $\theta \sigma_{S} \in \mathcal{L}^{2}$, i.e., we require that the following two integrability conditions hold $\mathbb{P}$-a.s.

$$
\int_{0}^{T}\left|c_{t}\right| d t<\infty, \quad \int_{0}^{T}\left(\theta_{t} \sigma_{S}(t)\right)^{2} d t<\infty .
$$

Under Assumption 2, we see that $\theta \sigma_{S} \in \mathcal{L}^{2}$ and Cauchy-Schwartz's inequality imply that $\theta \mu \in \mathcal{L}^{1}$ and, as a consequence, the wealth dynamics given by (5) is well-defined.

We place two additional requirements on the investor's possible choices. The first is the natural economic requirement that at the end of the time horizon, the investor has no remaining debt obligations and, thus, we require the processes $(c, \theta)$ to be such that

$$
\mathbb{P}\left(X_{T}^{(c, \theta)} \geq 0\right)=1
$$

As we shall see, the optimal strategies $(\hat{c}, \hat{\theta})$ have the property that $X_{T}^{(\hat{c}, \hat{\theta})}=0, \mathbb{P}$-almost surely. The second requirement is purely technical and is an artifact of our continuous- 
time setting: in order to provide rigorous proofs, we need a certain degree of regularity of the investor's possible choices. To state this regularity condition, we define the auxiliary deterministic function

$$
g(t)=\delta-r(t)+\frac{1}{2} \lambda_{S}(t)^{2}+a\left(\mu_{Y}(t)-\rho \sigma_{Y}(t) \lambda_{S}(t)\right)-\frac{1}{2}\left(1-\rho^{2}\right) a^{2} \sigma_{Y}(t)^{2},
$$

for $t \in[0, T]$. Furthermore, we define the function

$$
V(t, x, y)=-A(t) \exp \left\{-a A(t)^{-1} x-a y-A(t)^{-1} \int_{t}^{T} \beta(t, s) \int_{t}^{s} g(u) d u d s\right\},
$$

for $t \in[0, T]$ and $x, y \in \mathbb{R}$, which turns out to be the value function (see Theorem 1 below). We denote by $V_{t}, V_{x}$ and $V_{y}$ the respective partial derivatives of $V$. Finally, we can define the admissibility concept adopted in this paper as follows:

Definition 1. A pair $(c, \theta)$ of progressively measurable processes satisfying (6) and (7) as well as ensuring that the following stochastic integrals for $t \in[0, T]$

$$
\int_{0}^{t} e^{-\delta u} V_{x}\left(u, X_{u}^{(c, \theta)}, Y_{u}\right) \theta_{u} \sigma_{S}(u) d W_{u}, \quad \int_{0}^{t} e^{-\delta u} V_{y}\left(u, X_{u}^{(c, \theta)}, Y_{u}\right) \sigma_{Y}(u) d Z_{u}
$$

are genuine martingales, are deemed admissible. In this case, we write $(c, \theta) \in \mathcal{A}$.

It is a consequence of (6) and Itô's lemma that the stochastic integrals (10) are always well-defined local martingales. In order to rigorously prove our main existence result stated in the next section, we insist on only allowing the investor to use those strategies that produce genuine martingales in (10). For square integrable integrands, the stochastic integrals are indeed martingales (Protter 2004, Ch. IV.2).

\subsection{The optimal strategies}

The investor is assumed to maximize expected utility of consumption and therefore seeks a pair $(\hat{c}, \hat{\theta}) \in \mathcal{A}$ attaining the following maximum

$$
\sup _{(c, \theta) \in \mathcal{A}} \mathbb{E}\left[\int_{0}^{T}-e^{-\delta s} e^{-a c_{s}} d s\right], \quad X_{0}^{(c, \theta)}=x .
$$

We do not require the investor's strategies to produce finite expectation, i.e., we allow for strategies such that $\mathbb{E}\left[\int_{0}^{T} e^{-a c_{s}} d s\right]=+\infty$. Of course, the optimal strategies $(\hat{c}, \hat{\theta})$ produce a finite expectation.

As usual, in order to apply the dynamic programming principle, the problem is embedded 
into a family of problems, and since $\left(X^{(c, \theta)}, Y\right)$ form a Markovian system, we can define

$$
J(t, x, y)=\operatorname{esssup}_{(c, \theta) \in \mathcal{A}} \mathbb{E}_{t}\left[\int_{t}^{T}-e^{-\delta(s-t)} e^{-a c_{s}} d s\right], \quad t \in[0, T], \quad X_{t}^{(c, \theta)}=x, \quad Y_{t}=y
$$

We refer to $J$ as the value function or the indirect utility function.

The explicit solution to the investor's problem is stated in the following theorem, and the proof can be found in the Appendix.

Theorem 1. When Assumptions 1 and 2 are satisfied, the value function $J$ defined in (11) is identical to the function $V$ defined in (9), i.e., $J=V$. Furthermore, the optimal consumption and investment strategies are given by

$$
\begin{aligned}
& \hat{c}(t, x, y)=A(t)^{-1} x+y+\frac{1}{a} A(t)^{-1} \int_{t}^{T} \beta(t, s) \int_{t}^{s} g(u) d u d s \\
& \hat{\theta}(t, x, y)=A(t)\left(\frac{\lambda_{S}(t)}{a \sigma_{S}(t)}-\frac{\rho \sigma_{Y}(t)}{\sigma_{S}(t)}\right)
\end{aligned}
$$

for $x, y \in \mathbb{R}$ and $t \in[0, T]$, where the deterministic function $g$ is defined by (8).

As shown in the Appendix, the optimal strategies are such that the dynamics of the optimal financial wealth $\hat{X}_{t}=X_{t}^{(\hat{c}, \hat{\theta})}$ is given by

$$
d \hat{X}_{t}=\left[m(t)-\left(A(t)^{-1}-r(t)\right) \hat{X}_{t}\right] d t+A(t)\left[a^{-1} \lambda_{S}(t)-\rho \sigma_{Y}(t)\right] d W_{t},
$$

for the mean reversion level $m$ defined by

$$
m(t)=A(t) \lambda_{S}(t)\left[a^{-1} \lambda_{S}(t)-\rho \sigma_{Y}(t)\right]-a^{-1} A(t)^{-1} \int_{t}^{T} \beta(t, s) \int_{t}^{s} g(u) d u d s .
$$

In other words, the optimal financial wealth is an Ornstein-Uhlenbeck type process with time-dependent coefficients. As $t \rightarrow T$, the speed of mean reversion, $A(t)^{-1}-r(t)$, converges to infinity, while both $m(t)$ and the volatility of $\hat{X}$ converge to zero. As we prove in the Appendix, this forces the optimal terminal wealth to become zero at time $T$, i.e., we have $\hat{X}_{T}=0$ almost surely and, in particular, (7) is satisfied. In other words, the optimal financial wealth is given by an Ornstein-Uhlenbeck type bridge process.

\subsection{Discussion}

We can measure how the investor values the income stream by the extra initial wealth which is needed to compensate the investor for the loss of the entire income stream. For the case 
of no income at all, the value function is given by

$$
\bar{V}(t, x)=-A(t) \exp \left\{-a A(t)^{-1} x-A(t)^{-1} \int_{t}^{T} \beta(t, s) \int_{t}^{s} \bar{g}(u) d u d s\right\}
$$

where

$$
\bar{g}(t)=\delta-r(t)+\frac{1}{2} \lambda_{S}(t)^{2}=g(t)-a\left(\mu_{Y}(t)-\rho \sigma_{Y}(t) \lambda_{S}(t)-\frac{a}{2}\left(1-\rho^{2}\right) \sigma_{Y}(t)^{2}\right) .
$$

The wealth equivalent of the income stream, $L(t, y)$, is then defined by $\bar{V}(t, x+L(t, y))=$ $V(t, x, y)$, which implies that

$$
L(t, y)=A(t) y+\int_{t}^{T} \beta(t, s) \int_{t}^{s}\left(\mu_{Y}(u)-\rho \sigma_{Y}(u) \lambda_{S}(u)-\frac{a}{2}\left(1-\rho^{2}\right) \sigma_{Y}(u)^{2}\right) d u d s .
$$

The first term is the present value of a constant payment stream at the rate $y$, whereas the second term corrects for income growth, covariance with the stock price, and unspanned income risk. The sum of the two terms is equal to the expectation of the discounted future income under an appropriately risk-adjusted measure $\mathbb{Q}$. The measure $\mathbb{Q}$ is defined by applying the market-given price of risk $\lambda_{S}(t)$ for the risk represented by $W$ and the agent-specific price of risk $\lambda_{Y}(t)=\frac{a}{2} \sqrt{1-\rho^{2}} \sigma_{Y}(t)$ associated with the unspanned income risk represented by $Z$. The income dynamics can be written as

$$
d Y_{t}=\left(\mu_{Y}(t)-\rho \sigma_{Y}(t) \lambda_{S}(t)-\frac{a}{2}\left(1-\rho^{2}\right) \sigma_{Y}(t)^{2}\right) d t+\sigma_{Y}(t)\left(\rho d W_{t}^{\mathbb{Q}}+\sqrt{1-\rho^{2}} d Z_{t}^{\mathbb{Q}}\right),
$$

where $W^{\mathbb{Q}}$ and $Z^{\mathbb{Q}}$ are independent $\mathbb{Q}$-Brownian motions. This produces the expectation

$$
\mathbb{E}_{t}^{\mathbb{Q}}\left[\int_{t}^{T} \beta(t, s) Y_{s} d s\right]=L\left(t, Y_{t}\right)
$$

The value function (9) can be rewritten as

$$
V(t, x, y)=-A(t) \exp \left\{-a A(t)^{-1}[x+H(t)+L(t, y)]\right\}
$$

where

$$
H(t)=a^{-1} \int_{t}^{T} \beta(t, s) \int_{t}^{s}\left(\delta-r(u)+\frac{1}{2} \lambda_{S}(u)^{2}\right) d u d s
$$

can be interpreted as a correction of current wealth due to the future investment opportunities offered by the risk-free asset and the stock. The optimal consumption (12) can be expressed as

$$
\hat{c}(t, x, y)=A(t)^{-1}[x+H(t)+L(t, y)] .
$$


Hence, the optimal consumption at time $t$ is equal to the constant consumption the investor can get by "annuitizing" the perceived total wealth $X_{t}+H(t)+L\left(t, Y_{t}\right)$ over the remaining lifetime. As perceived total wealth changes and the remaining time period shortens, the optimal consumption changes. Note that the investor with initial wealth $x$ and the given income stream consumes exactly as an investor with initial wealth $x+L(t, y)$ and no income.

Given the dynamics of financial wealth in (14), the dynamics of the sum of financial wealth and the present value of future income is of the form

$$
d\left(\hat{X}_{t}+L\left(t, Y_{t}\right)\right)=\ldots d t+A(t) a^{-1} \lambda_{S}(t) d W_{t}+A(t) \sqrt{1-\rho^{2}} \sigma_{Y}(t) d Z_{t}
$$

Therefore, the optimal investment strategy with unspanned income risk is such that the total wealth has the same sensitivity towards the hedgeable shocks represented by $d W_{t}$ as the financial wealth has in the case without unspanned income risk. The presence of the unhedgeable shock represented by $d Z_{t}$ does not affect the desired exposure to the hedgeable shock. The dynamics of the optimal consumption $\hat{c}_{t}=\hat{c}\left(t, \hat{X}_{t}, Y_{t}\right)$ becomes

$$
d \hat{c}_{t}=\mu_{c}(t) d t+a^{-1} \lambda_{S}(t) d W_{t}+\sqrt{1-\rho^{2}} \sigma_{Y}(t) d Z_{t}
$$

for some deterministic function of time $\mu_{c}$. Note that any idiosyncratic income shock (positive or negative) leads to an immediate identical change in consumption.

Theorem 1 shows that the optimal number of stocks held by the investor is deterministic and, in particular, independent of wealth and income. The amount optimally invested in the stock at time $t$ is

$$
\hat{\theta}(t, x, y) S_{t}=A(t)\left(\frac{\lambda_{S}(t)}{a \sigma_{S}(t) / S_{t}}-\frac{\rho \sigma_{Y}(t)}{\sigma_{S}(t) / S_{t}}\right),
$$

where $\sigma_{S}(t) / S_{t}$ is the relative volatility of the stock. The optimal stock position is a combination of the standard myopic term and a term correcting for the stock-like component of the income process (an "income hedge" term), in accordance with the standard results for dynamic portfolio problems (Merton 1969, 1971; Bodie, Merton, and Samuelson 1992).

Henderson (2005) maximizes negative exponential utility of terminal wealth assuming a constant interest rate $r$, a constant Sharpe ratio $\lambda_{S}$, and a Gaussian income like (4) but with constant drift and volatility. Henderson finds that the amount optimally invested in the stock at time $t$ is ${ }^{12}$

$$
\theta^{\text {Hend }}(t, x, y) S_{t}=e^{-r(T-t)} \frac{\lambda_{S}}{a \sigma_{S} / S_{t}}-A(t) \frac{\rho \sigma_{Y}}{\sigma_{S} / S_{t}}
$$

\footnotetext{
${ }^{12}$ Henderson assumes that the relative volatility of the stock, $\sigma_{S} / S_{t}$, is constant, but this has no impact on the structure of the optimal portfolio. Our expression for the optimal portfolio generalizes to the case with any stochastic volatility as long as the risk-free rate and the Sharpe ratio of the stock are deterministic, see e.g., Detemple, Garcia, and Rindisbacher (2003) and Nielsen and Vassalou (2006).
} 
The appropriate weight on the speculative investment is $e^{-r(T-t)}$ with utility of terminal wealth, but $A(t)$ in our case with utility of intermediate consumption. In Henderson's case, the weight is the present value of getting a unit payment at the terminal date. In our case, the weight is the present value of getting a unit continuous payment over the remaining lifetime. Furthermore, note that if we let $T \rightarrow \infty$ our results become similar to those found in the infinite time horizon setting of Svensson and Werner (1993).

Finally, we consider the effect of increasing the magnitude of the unspanned component of the income risk, i.e., increasing $\sigma_{Y}(t) \sqrt{1-\rho^{2}}$. Since the function $g(t)$ in (8) then decreases, it is clear from (12) that optimal consumption is reduced. The reason is that the implicit valuation of the future income stream is lower, as can be seen from (16). Since the optimal investment in the stock is unaffected, the reduced consumption translates into a higher investment in the risk-free asset. This is the precautionary savings caused by risk aversion and unhedgeable persistent income shocks.

\section{Equilibrium}

\subsection{Derivation of the equilibrium}

We derive the equilibrium risk-free rate and stock price in the economy by aggregation over the investors. We let $\tau_{i}=1 / a_{i}$ denote the absolute risk tolerance of investor $i$ and let $\tau_{\Sigma}=\sum_{i=1}^{I} \tau_{i}$ denote the aggregate absolute risk tolerance in the economy.

As shown in Theorem 1, investor $i$ optimally invests in

$$
\hat{\theta}_{i}(t)=A(t)\left(\tau_{i} \frac{\lambda_{S}(t)}{\sigma_{S}(t)}-\frac{\rho_{i} \sigma_{Y i}(t)}{\sigma_{S}(t)}\right), \quad t \in[0, T]
$$

units of the stock. To ensure that the stock market clears, we need $\sum_{i} \hat{\theta}_{i}(t)=1$, which implies that the equilibrium Sharpe ratio of the stock (or the market price of risk) is

$$
\lambda_{S}(t)=\frac{1}{\tau_{\Sigma}}\left[A(t)^{-1} \sigma_{S}(t)+\sum_{i} \rho_{i} \sigma_{Y i}(t)\right],
$$

where the volatility $\sigma_{S}(t)$ is still to be identified.

The stock price $S$ is given as the appropriately risk-adjusted expectation of the discounted future dividends. We will show that the stock price defined as

$$
S_{t}=\mathbb{E}_{t}^{\mathbb{Q}^{\min }}\left[\int_{t}^{T} \beta(t, u) D_{u} d u\right], \quad t \in[0, T]
$$

is the equilibrium stock price. Here $\mathbb{Q}^{\text {min }}$ is the minimal martingale measure defined by the 
Radon-Nikodym derivative

$$
\frac{d \mathbb{Q}^{\text {min }}}{d \mathbb{P}}=\exp \left\{-\frac{1}{2} \int_{0}^{T} \lambda_{S}(t)^{2} d t-\int_{0}^{T} \lambda_{S}(t) d W_{t}\right\}
$$

This measure reflects that the adjustment for the risk affecting the dividend stream (1) is given by the market price of risk $\lambda_{S}(t)$, whereas the value of the discounted dividends is not affected by the idiosyncratic income shocks under our assumptions. The dividend dynamics (1) can be rewritten as

$$
d D_{t}=\left[\mu_{D}(t)-\lambda_{S}(t) \sigma_{D}(t)\right] d t+\sigma_{D}(t) d W_{t}^{\min }
$$

where $W_{t}^{\text {min }}=W_{t}+\int_{0}^{t} \lambda_{S}(u) d u$ defines a standard Brownian motion under $\mathbb{Q}^{\text {min }}$. By means of Fubini's theorem, we now find that

$$
\begin{aligned}
S_{t} & =\int_{t}^{T} \beta(t, u) \mathbb{E}_{t}^{\mathbb{Q}^{\min }}\left[D_{u}\right] d u \\
& =\int_{t}^{T} \beta(t, u)\left(D_{t}+\int_{t}^{u}\left(\mu_{D}(s)-\lambda_{S}(s) \sigma_{D}(s)\right) d s\right) d u \\
& =A(t) D_{t}+\int_{t}^{T} \beta(t, u) \int_{t}^{u}\left(\mu_{D}(s)-\lambda_{S}(s) \sigma_{D}(s)\right) d s d u .
\end{aligned}
$$

In particular, the (absolute) volatility of the stock price is $\sigma_{S}(t)=A(t) \sigma_{D}(t)$. By substituting this expression for $\sigma_{S}$ into (18), we obtain the following expression for the Sharpe ratio $\lambda_{S}(t)$ in terms of only exogenous quantities:

$$
\lambda_{S}(t)=\frac{1}{\tau_{\Sigma}}\left[\sigma_{D}(t)+\sum_{i} \rho_{i} \sigma_{Y i}(t)\right], \quad t \in[0, T] .
$$

Therefore, we see that Assumption 1 ensures that $\lambda_{S}$ given in (20) satisfies the second part of Assumption 2.

The bond market clearing condition is equivalent to the condition that the total financial wealth is invested in the stock, i.e., $S_{t}=\sum_{i} \hat{X}_{i t}$ for all $t \in[0, T]$ where $\hat{X}_{i}$ denotes investor $i$ 's optimal wealth process given by (14). We next show that $S$ given by (19) satisfies this relationship. Since $\hat{X}_{i T}=0$ for all $i$, it is immediate that the relation holds at $t=T$ and, hence, to show the validity at time $t, t<T$, it suffices to verify that $d S_{t}=\sum_{i} d \hat{X}_{i t}$. 
From (5) and (12), the optimal wealth dynamics of investor $i$ can be written as

$$
\begin{aligned}
d \hat{X}_{i t}= & \left(\hat{X}_{i t} r(t)+Y_{i t}-\hat{c}_{i t}\right) d t+\hat{\theta}_{i t} \mu_{S}(t) d t+\hat{\theta}_{i t} \sigma_{S}(t) d W_{t} \\
= & {\left[\left(r(t)-A(t)^{-1}\right) \hat{X}_{i t}-A(t)^{-1} \int_{t}^{T} \beta(t, s) \int_{t}^{s} \tau_{i} g_{i}(u) d u d s\right] d t } \\
& +\hat{\theta}_{i t} \mu_{S}(t) d t+\hat{\theta}_{i t} \sigma_{S}(t) d W_{t} .
\end{aligned}
$$

By summing up and using $\sum_{i} \hat{\theta}_{i t}=1$, we obtain

$$
\begin{aligned}
\sum_{i} d \hat{X}_{i t}=[ & \left(r(t)-A(t)^{-1}\right) \sum_{i} \hat{X}_{i t}+\mu_{S}(t) \\
& \left.-A(t)^{-1} \int_{t}^{T} \beta(t, s) \int_{t}^{s}\left(\sum_{i} \tau_{i} g_{i}(u)\right) d u d s\right] d t+\sigma_{S}(t) d W_{t}
\end{aligned}
$$

We rewrite the stock price dynamics (2) as

$$
d S_{t}=\left[\left(r(t)-A(t)^{-1}\right) S_{t}+\mu_{S}(t)+A(t)^{-1} S_{t}-D_{t}\right] d t+\sigma_{S}(t) d W_{t}
$$

to see that $d S_{t}=\sum_{i} d \hat{X}_{i t}$ if, and only if,

$$
S_{t}=A(t) D_{t}-\int_{t}^{T} \beta(t, s) \int_{t}^{s}\left(\sum_{i} \tau_{i} g_{i}(u)\right) d u d s
$$

By comparing (19) and (21), we conclude that (21) is equivalent to

$$
\mu_{D}(t)-\lambda_{S}(t) \sigma_{D}(t)=-\sum_{i} \tau_{i} g_{i}(t), \quad t \in[0, T]
$$

Therefore, by substituting in $g_{i}(t)$ from (8) and rearranging, we find

$$
\begin{aligned}
r(t) \tau_{\Sigma}= & \sum_{i} \tau_{i} \delta_{i}+\mu_{D}(t)+\sum_{i} \mu_{Y i}(t) \\
& -\lambda_{S}(t)\left[\sigma_{D}(t)+\sum_{i} \rho_{i} \sigma_{Y i}(t)-\frac{1}{2} \tau_{\Sigma} \lambda_{S}(t)\right]-\frac{1}{2} \sum_{i} a_{i}\left(1-\rho_{i}^{2}\right) \sigma_{Y i}(t)^{2} .
\end{aligned}
$$

Finally, the expression (20) for $\lambda_{S}(t)$ can be used. We summarize our findings in the following theorem. 
Theorem 2. Under Assumption 1, the equilibrium risk-free rate is

$$
\begin{aligned}
r(t)= & \sum_{i} \frac{\tau_{i}}{\tau_{\Sigma}} \delta_{i}+\frac{1}{\tau_{\Sigma}}\left[\mu_{D}(t)+\sum_{i} \mu_{Y i}(t)\right] \\
& -\frac{1}{2 \tau_{\Sigma}^{2}}\left[\sigma_{D}(t)+\sum_{i} \rho_{i} \sigma_{Y i}(t)\right]^{2}-\frac{1}{2 \tau_{\Sigma}^{2}} \sum_{i} \frac{\tau_{\Sigma}}{\tau_{i}}\left(1-\rho_{i}^{2}\right) \sigma_{Y i}(t)^{2},
\end{aligned}
$$

and the equilibrium stock price $S_{t}$ is

$$
S_{t}=A(t) D_{t}+\int_{t}^{T} \beta(t, u) \int_{t}^{u}\left(\mu_{D}(s)-\tau_{\Sigma} \sigma_{D}(s)\left[\sigma_{D}(s)+\sum_{i} \rho_{i} \sigma_{Y i}(s)\right]\right) d s d u
$$

This leads to a stock price volatility of $\sigma_{S}(t)=A(t) \sigma_{D}(t)$ and the Sharpe ratio

$$
\lambda_{S}(t)=\frac{1}{\tau_{\Sigma}}\left[\sigma_{D}(t)+\sum_{i} \rho_{i} \sigma_{Y i}(t)\right], \quad t \in[0, T] .
$$

In particular, $r(t)$ satisfies the first part and $\lambda_{S}(t)$ the second part of Assumption 2.

We compare our equilibrium risk-free rate and Sharpe ratio to a dynamically complete market economy in the following subsection, but first we highlight some aspects of our equilibrium.

The Sharpe ratio increases when more of the income risk is spanned, either due to higher income-stock correlations $\rho_{i}$ or higher income volatilities $\sigma_{Y i}$ (or both). Higher $\rho_{i} \sigma_{Y i}$ leads to a larger (negative) income hedge demand for the stock, cf. (17), and, consequently, a lower total stock demand. To maintain market clearing, a higher Sharpe ratio is necessary in order to increase the speculative demand. The investors' risk attitudes determine how much the Sharpe ratio has to increase. An increase in $\rho_{i} \sigma_{Y i}$ does not affect the absolute volatility of the stock, but it lowers the stock price and, thus, increases the relative volatility $\sigma_{S}(t) / S_{t}$. The expected excess rate of return on the stock, $\mu_{S}(t) / S_{t}=\lambda_{S}(t) \sigma_{S}(t) / S_{t}$, is therefore also increasing in $\rho_{i} \sigma_{Y i}$.

The equilibrium risk-free rate is determined by the risk-tolerance weighted average of time preference rates, the expected aggregate consumption growth divided by the aggregate risk tolerance, and two risk adjustment terms. The first of these terms adjusts for spanned aggregate $W$-risk and is the same as in a complete market (see below). The second risk adjustment term relates to aggregate unspanned income risk and differs from the first adjustment term via the presence of the ratio $\tau_{\Sigma} / \tau_{i}$ in the sum. Hence, the unspanned income risk has a different effect on the equilibrium risk-free rate than the spanned income risk. For simplicity, assume for a moment that $a_{i}=a=1 / \tau, \rho_{i}=\rho$, and $\sigma_{Y i}(t)=\sigma_{Y}(t)$ for all $I$ investors, and let $\bar{\sigma}_{D}(t)=\sigma_{D}(t) / I$ be the absolute volatility of per capita dividends. In this 
case, the last two terms in (22) add up to $-\frac{1}{2 \tau^{2}}\left[\sigma_{Y}(t)^{2}+\bar{\sigma}_{D}(t)^{2}+2 \rho \bar{\sigma}_{D}(t) \sigma_{Y}(t)\right]$, which is decreasing in the income-dividend correlation. Intuitively, a higher income-dividend correlation induces investors to shift funds from the risky to the risk-free asset and, therefore, in order to maintain market clearing, the equilibrium risk-free rate has to decrease.

Finally, the equilibrium provided by Theorem 2 constitutes a setting in which all European claims written on the stock are replicable by continuous trading in the money market as well as the stock itself. However, the resulting market is incomplete because each investor's income process $Y_{i}$ cannot be fully hedged due to the presence of the idiosyncratic risk component $Z_{i}$. As we shall see in the next section, the equilibrium market is not Pareto efficient because there is a discrepancy relative to the dynamically complete market equilibrium. By the second welfare theorem the equilibrium is therefore not Pareto efficient.

\subsection{Comparison with a dynamically complete market equilibrium}

Aggregate consumption in our economy is $C_{\Sigma t}=\sum_{i} \hat{c}_{i t}=D_{t}+\sum_{i} Y_{i t}$ with dynamics

$$
d C_{\Sigma t}=\left[\mu_{D}(t)+\sum_{i} \mu_{Y i}(t)\right] d t+\left[\sigma_{D}(t)+\sum_{i} \rho_{i} \sigma_{Y i}(t)\right] d W_{t}+\sum_{i} \sqrt{1-\rho_{i}^{2}} \sigma_{Y i}(t) d Z_{i t}
$$

In order to get a better understanding of the impact from unspanned income risk, we compare our equilibrium to the equilibrium in an economy with the same aggregate consumption dynamics and same preferences, but where all income risks are spanned through the existence of financial securities facilitating a dynamically complete market, cf. Duffie and Huang (1985). ${ }^{13}$ Market completeness ensures the existence of a unique state-price density process $\xi_{t}^{\mathrm{COMP}}$ with dynamics

$$
d \xi_{t}^{\mathrm{COMP}}=-\xi_{t}^{\mathrm{COMP}}\left[r_{t}^{\mathrm{COMP}} d t+\lambda_{W t}^{\mathrm{COMP}} d W_{t}+\sum_{i} \lambda_{Z_{i} t}^{\mathrm{COMP}} d Z_{i t}\right], \quad \xi_{0}^{\mathrm{COMP}}=1
$$

where $r_{t}^{\mathrm{COMP}}$ is the equilibrium risk-free rate, and where $\lambda_{W t}^{\mathrm{COMP}}$ and $\lambda_{Z_{i} t}^{\mathrm{COMP}}$ are the market prices of risk associated with the different shocks.

Because our focus here is on the asset prices (and not the trading strategies of the agents), it suffices to consider the Arrow-Debreu equilibrium in which each agent $i$ maximizes expected

\footnotetext{
${ }^{13}$ In our setting with a finite set of investors, completeness can be obtained with a finite set of financial securities spanning the individual income risks, such as long-lived insurance contracts. Obviously, there are many good reasons why such contracts cannot exist in actual markets, such as concerns for moral hazard, adverse selection, and potential fraud associated with labor income risks.
} 
life-time utility $\mathbb{E}\left[\int_{0}^{T} U_{i}\left(t, c_{t}\right) d t\right]$ subject to the static budget constraint

$$
\mathbb{E}\left[\int_{0}^{T} \xi_{t}^{\mathrm{COMP}} c_{i t} d t\right] \leq x_{i}
$$

where $x_{i}$ denotes investor $i$ 's initial wealth. The first-order condition for the optimal time $t$ consumption $\hat{c}_{i t}^{\mathrm{COMP}}$ is $\frac{\partial U_{i}}{\partial c}\left(t, \hat{c}_{i t}^{\mathrm{COMP}}\right)=y_{i} \xi_{t}^{\mathrm{COMP}}, t \in[0, T]$, where $y_{i}>0$ is the Lagrange multiplier for the budget constraint (26). Solving for $\hat{c}_{i t}^{\text {COMP }}$ and aggregating over agents, we find

$$
C_{\Sigma t}=\sum_{i} \hat{c}_{i t}^{\mathrm{COMP}}=-\left(\sum_{i} \tau_{i} \delta_{i}\right) t-\sum_{i} \tau_{i} \ln \left(\tau_{i} y_{i}\right)-\tau_{\Sigma} \ln \left(\xi_{t}^{\mathrm{COMP}}\right)
$$

By applying Itô's lemma to $C_{\Sigma t}$ and matching the resulting dynamics to (25), we find the market price of risk processes to be

$$
\begin{aligned}
& \lambda_{W t}^{\mathrm{COMP}}=\frac{1}{\tau_{\Sigma}}\left[\sigma_{D}(t)+\sum_{i} \rho_{i} \sigma_{Y i}(t)\right] \\
& \lambda_{Z_{i} t}^{\mathrm{COMP}}=\frac{1}{\tau_{\Sigma}} \sqrt{1-\rho_{i}^{2}} \sigma_{Y i}(t), \quad i \in\{1,2, \ldots, I\} .
\end{aligned}
$$

For the aggregate consumption dynamics (25), the deterministic complete market Sharpe ratio $\lambda_{W}^{\mathrm{COMP}}$ in (27) is identical to the equilibrium incomplete market Sharpe ratio $\lambda_{S}$ given by (24). Therefore, the incompleteness caused by unspanned income risk does not affect the equilibrium market price of spanned risk. In the complete market setting, both $W$-risk and the idiosyncratic income risks are efficiently shared among the investors and, thus, the market prices of idiosyncratic income risks $\lambda_{Z_{i}}^{\text {COMP }}$ in (28) are also determined by the aggregate risk tolerance in the economy.

Similarly, we find the equilibrium risk-free rate process to be

$$
\begin{aligned}
r_{t}^{\mathrm{COMP}}= & \sum_{i} \frac{\tau_{i}}{\tau_{\Sigma}} \delta_{i}+\frac{1}{\tau_{\Sigma}}\left[\mu_{D}(t)+\sum_{i} \mu_{Y i}(t)\right] \\
& -\frac{1}{2 \tau_{\Sigma}^{2}}\left(\left[\sigma_{D}(t)+\sum_{i} \rho_{i} \sigma_{Y i}(t)\right]^{2}+\sum_{i}\left(1-\rho_{i}^{2}\right) \sigma_{Y i}(t)^{2}\right)
\end{aligned}
$$

This expression shows that the equilibrium risk-free rate is highly affected by the incompleteness. In the absence of idiosyncratic income risk, i.e., when $\rho_{i}^{2}=1$ for all investors $i$, the two expressions for the risk-free rate (22) and (29) are identical. The two first terms in the expressions (22) and (29) are identical and, thus, not affected by unspanned income risk. The first term is a risk-tolerance weighted average of the individual time preference rates consistent with Gollier and Zeckhauser (2005). The second term in the risk-free rate is the product of the expected growth rate of aggregate consumption and the reciprocal of the 
aggregate risk tolerance. Obviously, independent increments in the aggregate consumption process is a key requirement for a deterministic equilibrium risk-free rate both in the complete and the incomplete market setting. The unspanned income risk affects the equilibrium risk-free rate via the precautionary savings motive as reflected by the difference in the last term on the right-hand sides of (22) and (29). Risk-averse investors exposed to unhedgeable income shocks increase their demand for the risk-free asset, which leads to a higher equilibrium price and a lower equilibrium risk-free rate. Other things being equal, more risk-averse investors increase their demand for the risk-free asset by more. Therefore, in determining the equilibrium risk-free rate it is natural that the unspanned income risk of each investor is scaled by the investor's personal risk aversion. Indeed, this is reflected by our solution for the optimal consumption and investment strategies as discussed in the final paragraph of Section 3. With identical aggregate consumption, the difference between the risk-free rate in the economy with unspanned income risk and in the dynamically complete market economy is

$$
r(t)-r_{t}^{\mathrm{COMP}}=-\frac{1}{2 \tau_{\Sigma}^{2}} \sum_{i}\left(\frac{\tau_{\Sigma}}{\tau_{i}}-1\right)\left(1-\rho_{i}^{2}\right) \sigma_{Y i}(t)^{2}
$$

Since $\tau_{\Sigma}=\sum_{i} \tau_{i}>\tau_{j}$ for any $j$ if $I \geq 2$, we have the following corollary to Theorem 2 :

Corollary 1. Under Assumption 1 the following holds. If $I \geq 2$ and $\sigma_{Y i}(t) \neq 0$ and $\rho_{i}^{2}<1$ for at least one $i$, the equilibrium risk-free rate in the economy with unspanned income risk is smaller than the equilibrium risk-free rate in the corresponding complete market economy with identical aggregate consumption dynamics.

The equilibrium risk-free rate is lower with unspanned income risk than in an otherwise identical complete market economy due to the fact that investors cannot efficiently share income risk but only self-insure through precautionary savings in the risk-free asset. This is reflected in (30) by the term $\tau_{\Sigma} / \tau_{i}-1$ : in equilibrium, the investors evaluate their future unspanned income risk, $Z_{i}$, using their personal risk tolerance $\tau_{i}$ as opposed to the aggregate risk tolerance in the economy $\tau_{\Sigma}$ as they are using for evaluating $W$-risks which can be efficiently shared in the market. Our paper is the first to solve in closed-form for the equilibrium risk-free rate and the equilibrium stock price in a setting with a finite set of heterogeneous investors as well as unspanned income risk both at the individual and aggregate level. Furthermore, it suggests and explicitly quantifies the role of unspanned income risk in the determination of the equilibrium risk-free rate and as a potential explanation of the risk-free rate puzzle. With a lower risk-free rate and the same Sharpe ratio, it is clear that the expected return on the stock - keeping the volatility fixed - is also lower in our incomplete market setting than in an otherwise identical complete market setting with identical aggregate consumption dynamics. In turn, this implies that the equilibrium stock price is higher in the incomplete market setting than in the complete market setting. 


\subsection{Numerical example}

Our closed-form solution for the incomplete market equilibrium allows for straightforward comparative statics in terms of exogenous parameters. Moreover, the closed-form equilibrium allows us to quantify the impact of market incompleteness on equilibrium securities prices. In order to assess this quantitative impact of unspanned income risk, we start by assuming that all $I$ investors have the same absolute risk aversion $a_{i}=a$, the same constant income volatility $\sigma_{Y i}(t)=\sigma_{Y}$, and the same income-stock correlation $\rho_{i}=\rho$. Then $\tau_{\Sigma}=I / a$, and (30) reduces to

$$
r(t)-r_{t}^{\mathrm{COMP}}=-\frac{1}{2} a^{2}\left(1-\frac{1}{I}\right)\left(1-\rho^{2}\right) \sigma_{Y}^{2}, \quad t \in[0, T]
$$

This interest rate difference is increasing in the number of investors and by considering a limit economy with the number of investors going to infinity we find

$$
\lim _{I \rightarrow \infty}\left(r(t)-r_{t}^{\mathrm{COMP}}\right)=-\frac{1}{2} a^{2}\left(1-\rho^{2}\right) \sigma_{Y}^{2}, \quad t \in[0, T] .
$$

To evaluate this difference, we need estimates of $a, \rho$, and $\sigma_{Y}$. Various studies report that individuals typically seem to have a relative risk aversion around 2 (see, e.g., Szpiro 1986; Guo and Whitelaw 2006; Attanasio and Paiella 2011), labor income with a relative volatility of around 10\%, and a correlation with the stock market near zero (see, e.g., Gourinchas and Parker 2002; Cocco, Gomes, and Maenhout 2005; Davis, Kubler, and Willen 2006). If we assume that initial consumption and income are identical and represent future values in units of initial consumption, then the initial absolute and relative risk aversions are identical and the initial absolute and relative income volatilities are also identical. Due to this scaling conversion, we will therefore consider values of $a$ around 2 and values of $\sigma_{Y}$ around 0.1 (of course, with constant $a$ and $\sigma_{Y}$, a positive drift in consumption and income will imply an increasing relative risk aversion and a decreasing relative income volatility over life). With $a=2, \sigma_{Y}=0.1$, and $\rho=0$, the above limiting interest rate difference equals $-0.5 \cdot 2^{2}(1-$ $\left.0^{2}\right) 0.1^{2}=-0.02$. In other words, the model with idiosyncratic income risk produces a riskfree rate which is 2 percentage points lower than in the otherwise identical complete market economy. This difference is increasing in and highly dependent on the risk aversion level and the income volatility level, but less dependent on the income-stock correlation (as long as it is near zero). For the risk aversion $a=3$ (and keeping $\sigma_{Y}=0.1$ ), the risk-free rate reduction is 4.5 percentage points. Similarly, increasing the income volatility to $\sigma_{Y}=0.2$ (and keeping $a=2$ ) produces a risk-free rate reduction of 8 percentage points.

In order to assess the quantitative impact of the cross-sectional distribution of risk aversion and unspanned income volatility, we divide the population into two groups: a group $L$ of 


\begin{tabular}{lllll}
\hline & \multicolumn{4}{c}{$\left(a_{L}, a_{H}\right)$} \\
\cline { 2 - 5 }$w$ & $(2,2)$ & $(2,3)$ & $(3,2)$ & $(3,3)$ \\
\hline 1.00 & 0.0200 & 0.0200 & 0.0450 & 0.0450 \\
0.75 & 0.0350 & 0.0491 & 0.0567 & 0.0788 \\
0.50 & 0.0500 & 0.0840 & 0.0660 & 0.1125 \\
0.25 & 0.0650 & 0.1267 & 0.0736 & 0.1463 \\
0.00 & 0.0800 & 0.1800 & 0.0800 & 0.1800 \\
\hline
\end{tabular}

Table 1: Reduction in equilibrium risk-free rate due to incompleteness in the limiting case $(I \rightarrow \infty)$ for various weights $w$ [rows] and various degrees of risk aversions $\left(a_{L}, a_{H}\right)$ [columns]. The exogenous parameters are given by $\rho_{i}=0, \sigma_{L Y}=0.1$ and $\sigma_{H Y}=0.2$.

investors having the same relatively low income volatility $\sigma_{L Y}$ and a group $H$ of investors having the same relatively high income volatility $\sigma_{H Y}$. The degree of risk aversion is the same for all investors within each group ( $a_{L}$ and $a_{H}$, respectively), but may vary across groups. For simplicity, we assume that the income processes $Y_{i}$ are all completely unspanned, i.e., $\rho_{i}=0$ for all $i$. In the limiting case $I \rightarrow \infty$, the interest rate differential (30) becomes

$$
\lim _{I \rightarrow \infty}\left(r(t)-r_{t}^{\mathrm{COMP}}\right)=-\frac{1}{2\left(\frac{1-w}{a_{H}}+\frac{w}{a_{L}}\right)}\left((1-w) a_{H} \sigma_{H Y}^{2}+w a_{L} \sigma_{L Y}^{2}\right), \quad t \in[0, T] .
$$

Table 1 reports this limiting interest rate difference for various combinations of risk aversions $\left(a_{L}, a_{H}\right)$ as well as various population distributions $(w)$. First, the table confirms that the equilibrium risk-free rate can be substantially lower in our incomplete market model relative to the comparable complete market model. Second, we see that the equilibrium risk-free rate depends on the allocation of unspanned income risk across investors with heterogenous risk aversion, i.e., our model does not allow aggregation. When the two investor groups are of the same relative size $(w=0.5)$, the reduction in the risk-free rate is highest when the most risk-averse investors face the largest income risks, i.e., 8.4 percentage points as opposed 6.6 percentage points when the most risk-tolerant investors hold the largest income risk.

\section{Relation to equilibrium with heterogeneous expectations}

In this section we will briefly relate our results to the literature on heterogeneous expectations, i.e., when the investors have different subjective probability measures $\mathbb{P}_{i}, i=1,2 \ldots, I$. We 
define investor $i$ 's subjective probability measure $\mathbb{P}_{i}$ by

$$
\frac{d \mathbb{P}_{i}}{d \mathbb{P}}=M_{i T}, \quad d M_{i t}=-M_{i t} a_{i} \sigma_{i Y}\left(\rho_{i} d W_{t}+\sqrt{1-\rho_{i}^{2}} d Z_{i t}\right), \quad t \in[0, T], \quad M_{i 0}=1 .
$$

Girsanov's theorem ensures that $W_{i t}=W_{t}+a_{i} \sigma_{i Y} \rho_{i} t$ is a Brownian motion under $\mathbb{P}_{i}$. For $(c, \theta) \in \mathcal{A}$, we define $\tilde{c}_{t}=c_{t}-Y_{i t}$ and rewrite the wealth dynamics (5) as

$$
\begin{aligned}
d X_{i t} & =\left(r(t) X_{i t}+\theta_{t} \sigma_{S}(t) \lambda_{S}(t)-\tilde{c}_{t}\right) d t+\theta_{t} \sigma_{S}(t) d W_{t} \\
& =\left(r(t) X_{i t}+\theta_{t} \sigma_{S}(t) \lambda_{i S}(t)-\tilde{c}_{t}\right) d t+\theta_{t} \sigma_{S}(t) d W_{i t}
\end{aligned}
$$

where $\lambda_{i S}(t)=\lambda_{S}(t)-a_{i} \sigma_{i Y} \rho_{i}$ is investor $i$ 's subjective Sharpe ratio. We also define the investors' adjusted subjective time preference rate as

$$
\tilde{\delta}_{i}(t)=\delta_{i}+a_{i}\left(\frac{Y_{i 0}}{t}+\mu_{i Y}-\frac{a_{i}}{2 t} \sigma_{i Y}^{2}\right), \quad t \in[0, T], \quad i=1, \ldots, I .
$$

Using the above notation, we can then use Fubini's theorem to rewrite the individual investor's objective from Section 3.2 as

$$
\mathbb{E}\left[\int_{0}^{T}-e^{-\delta_{i} u-a_{i} c_{u}} d u\right]=\mathbb{E}^{\mathbb{P}_{i}}\left[\int_{0}^{T}-e^{-\tilde{\delta}_{i}(u) u-a_{i} \tilde{c}_{u}} d u\right] .
$$

As a consequence, the equilibrium we constructed in Theorem 2 can be seen as an equilibrium in which the unspanned income $Y_{i}$ is replaced by the heterogeneous expectations $\mathbb{P}_{i}$ and the specific time-dependent subjective time preference rates $\tilde{\delta}_{i}$ defined above. The market clearing conditions for the financial assets remain the same; however, the clearing condition for the consumption good becomes $\sum_{i} \hat{\tilde{c}}_{i t}=D_{t}$, where $\hat{\tilde{c}}_{i}$ denotes investor $i$ 's optimal consumption plan.

It might appear that rewriting our equilibrium problem in terms of heterogeneous expectations without unspanned income risk produces a standard Pareto efficient equilibrium. However, this is not the case. Cuoco and He (1994) introduce a representative agent framework with stochastic weighting which covers the heterogeneous setting presented above. Cuoco and He (1994) demonstrate that for non-constant weighting the resulting equilibrium is in general not Pareto efficient. The key is that the stock and the risk-free asset are not sufficient to facilitate the Pareto efficient side-betting, which follows from investors' heterogenous beliefs (see, e.g., Wilson 1968). 


\section{Conclusion}

This paper has offered the first closed-form solution for the equilibrium risk-free rate and the equilibrium stock price in a dynamic economy with a finite set of heterogeneous investors and unspanned income risk both at the individual and the aggregate level. The degree of spanning of the individual income risks has a clear impact on the equilibrium consumption and asset prices. A smaller fraction of income risk being spanned produces a lower equilibrium risk-free rate and a lower stock market Sharpe ratio, partly due to changes in aggregate consumption dynamics.

On the other hand, if we keep the aggregate consumption dynamics fixed, the Sharpe ratio is the same whereas the risk-free rate and expected stock return are both lower in the economy with unspanned income risk than an otherwise identical dynamically complete market setting with all income risks being spanned. The reduction in the risk-free rate depends on the magnitude of all investors' unspanned income risk as well as their risk aversion coefficients, and the reduction is highest when the more risk-averse investors face the largest unspanned income risk. In a numerical example with reasonable parameters, we illustrated that the reduction in the risk-free rate - and, thus, the expected stock return for a fixed volatilitycan be several percentage points. Our model therefore suggests that unspanned income risk may play an important role in explaining the risk-free rate puzzle.

The expected changes in labor income and the volatility of these changes are likely to change over the life cycle for the individual consumers-investors. Life-cycle profiles for labor income are easily encompassed in our model through the deterministic functions $\mu_{Y i}$ and $\sigma_{Y i}$ in the individuals' income processes. An unappealing feature, however, of our model for analyzing general equilibrium implications of life-cycle income profiles is that all individuals live on the common finite time interval $[0, T]$. On the other hand, having solved the finitelylived individuals' consumption-portfolio problem in closed-form, and derived the equilibrium, also in closed-form, by simple addition of their net-demands and imposing the market clearing conditions, it is straightforward to extend the model into an overlapping-generations model. The challenges are to model how new individuals enter into the economy, and at each date keeping track of the size and composition of the population in terms of expected changes in labor income and income volatility.

Similarly, we have assumed that the labor income processes are exogenous. It would be more appropriate to model these processes endogenously through the individuals' choice of, for example, education and occupation. This would require a model of the choice set specifying the relation between expected labor income and its volatility. Given an appropriate choice set (with a positive relation between expected income and its volatility), and our closed-form solution for the individuals' consumption-portfolio problem, it is straightforward to determine 
the individuals' optimal choice of the income process. The more risk-tolerant individuals would choose income processes with higher volatility relative to more risk-averse individuals. Hence, the reduction in the equilibrium risk-free rate compared to a complete market setting will be smaller than in an otherwise identical economy in which all individuals have the same (average) income volatility, cf. Section 4.3. Obviously, an overlapping-generations model with endogenous labor income processes would also include an endogenous dividend process for the stock market through the choice of production technologies. We leave these extensions of our model for future research.

Admittedly, our assumptions of negative exponential utility and normally distributed incomes and dividends may seem unrealistic. However, it seems impossible to obtain closedform solutions for both the investors' consumption-portfolio problems and the equilibrium securities prices using more appropriate preferences and income and dividend processes without imposing other questionable assumptions.

\section{A Proof of Theorem 1}

The Hamilton-Jacobi-Bellman (HJB) equation associated with the problem (11) is

$$
\begin{aligned}
\delta V=\sup _{c, \theta \in \mathbb{R}}\{ & -e^{-a c}+V_{t}+V_{x}\left\{r(t) x-c+y+\theta \mu_{S}(t)\right\}+\frac{1}{2} V_{x x} \theta^{2} \sigma_{S}(t)^{2} \\
& \left.+V_{y} \mu_{Y}(t)+\frac{1}{2} V_{y y} \sigma_{Y}(t)^{2}+V_{x y} \theta \rho \sigma_{S}(t) \sigma_{Y}(t)\right\}, \quad t \in[0, T), \quad x, y \in \mathbb{R},
\end{aligned}
$$

with subscripts on $V$ indicating partial derivatives. Since our investor only receives utility of running consumption, the terminal condition is given by

$$
V(T, x, y)=0, \quad x \geq 0, \quad y \in \mathbb{R},
$$

where the requirement $x \geq 0$ is due to our admissibility requirement (7). The proof proceeds through the usual steps required in order to apply the dynamic programming principle.

Step I: Explicit solution to the HJB equation. The first-order conditions in the HJB equation lead to the optimal control candidates

$$
\hat{c}_{t}=-a^{-1} \log \left(a^{-1} V_{x}\right), \quad \hat{\theta}_{t}=-\frac{V_{x}}{V_{x x}} \frac{\mu_{S}(t)}{\sigma_{S}(t)^{2}}-\frac{V_{x y}}{V_{x x}} \frac{\rho \sigma_{Y}(t)}{\sigma_{S}(t)} .
$$


By substituting these expressions into the HJB equation, we obtain the PDE

$$
\begin{aligned}
\delta V= & V_{t}+V_{x}\left(r(t) x+y-a^{-1}\right)+a^{-1} V_{x} \log \left(a^{-1} V_{x}\right)-\frac{1}{2} \frac{V_{x}^{2}}{V_{x x}} \lambda_{S}(t)^{2} \\
& -\frac{1}{2} \frac{V_{x y}^{2}}{V_{x x}} \rho^{2} \sigma_{Y}(t)^{2}+V_{y} \mu_{Y}(t)+\frac{1}{2} V_{y y} \sigma_{Y}(t)^{2}-\frac{V_{x} V_{x y}}{V_{x x}} \rho \sigma_{Y}(t) \lambda_{S}(t), t \in[0, T), x, y \in \mathbb{R} .
\end{aligned}
$$

We try a solution of the form $V(t, x, y)=-\exp \{-H(t) x-G(t) y-F(t)\}$ for smooth deterministic functions $F, G$ and $H$. The optimal control candidates are

$$
\begin{aligned}
& \hat{c}_{t}=-a^{-1}\left(\log \left(a^{-1} H(t)\right)-H(t) x-G(t) y-F(t)\right), \\
& \hat{\theta}_{t}=\frac{1}{H(t) \sigma_{S}(t)}\left(\lambda_{S}(t)-\rho \sigma_{Y}(t) G(t)\right) .
\end{aligned}
$$

Inserting these expressions into the PDE gives us the wealth dynamics (see (5))

$$
\begin{aligned}
d X_{t}^{(\hat{c}, \hat{\theta})} & =\left(X_{t}^{(\hat{c}, \hat{\theta})}\left\{r(t)-a^{-1} H(t)\right\}+Y_{t}\left\{1-a^{-1} G(t)\right\}+a^{-1}\left(\log \left(a^{-1} H(t)\right)-F(t)\right)\right. \\
& +\frac{\lambda_{S}(t)}{H(t)}\left(\lambda_{S}(t)-G(t) \rho \sigma_{Y}(t)\right) d t+\frac{1}{H(t)}\left(\lambda_{S}(t)-\rho \sigma_{Y}(t) G(t)\right) d W_{t} .
\end{aligned}
$$

Based on the below lemma, we see that to ensure admissibility of the optimal control candidates, we wish to have

$$
\lim _{t \rightarrow T} H(t)=+\infty, \quad \lim _{t \rightarrow T} G(t)=a, \quad \lim _{t \rightarrow T} m(t)=\lim _{t \rightarrow T} \frac{1}{H(t)}\left(\rho \sigma_{Y}(t) G(t)-\lambda_{S}(t)\right)=0,
$$

where we have defined the mean reversion function $m$ by

$$
m(t)=a^{-1}\left(\log \left(a^{-1} H(t)\right)-F(t)\right)+\frac{\lambda_{S}(t)}{H(t)}\left(\lambda_{S}(t)-G(t) \rho \sigma_{Y}(t)\right), \quad t \in[0, T] .
$$

To compute $F, G$ and $H$ explicitly, we compute the involved derivatives and insert them into the above PDE. After dividing through with the exponential term, we collect the $x$ and $y$-terms to obtain the following coupled system of ODEs

$$
\begin{array}{lll}
x \text {-terms : } & H^{\prime}(t)=a^{-1} H(t)^{2}-H(t) r(t), \quad H(T)=+\infty, \\
y \text {-terms : } & G^{\prime}(t)=a^{-1} H(t) G(t)-H(t), & G(T)=a .
\end{array}
$$

Direct calculations show that $G(t)=a$ for $t \in[0, T]$ whereas the Ricatti equation for $H$ has the solution $H(t)=a A(t)^{-1}, t \in[0, T]$, where $A$ is the annuity factor. To get the terminal 
condition for $F$, we insert these expressions into the mean reversion function $m$

$$
m(t)=-a^{-1}(\log (A(t))+F(t))+A(t) \lambda_{S}(t)\left(a^{-1} \lambda_{S}(t)-\rho \sigma_{Y}(t)\right) . \quad t \in[0, T],
$$

From this expression, it follows that F's terminal condition is

$$
\lim _{t \rightarrow T}(\log (A(t))+F(t))=0
$$

Since $A(t) \rightarrow 0$ for $t \rightarrow T$, it must be the case that $F(t) \rightarrow \infty$ for $t \rightarrow T$, and this implies that the boundary condition (31) is satisfied for $x \geq 0$ and $y \in \mathbb{R}$. To get the explicit form for $F$, we collect the remaining terms of the PDE and find the linear ODE

$$
F^{\prime}(t)=A(t)^{-1}[\log (A(t))+1+F(t)]-g(t)-r(t)
$$

where we have defined the deterministic function

$$
g(t)=\delta-r(t)+\frac{1}{2} \lambda_{S}(t)^{2}+a\left(\mu_{Y}(t)-\rho \sigma_{Y}(t) \lambda_{S}(t)\right)-\frac{1}{2} a^{2}\left(1-\rho^{2}\right) \sigma_{Y}(t)^{2}, \quad t \in[0, T] .
$$

By using Leibnitz's rule, we can verify that $F$ is given by

$$
F(t)=\int_{t}^{T} e^{-\int_{t}^{s} A(u)^{-1} d u} g(s) d s-\log (A(t))=\tilde{F}(t)-\log (A(t)) .
$$

Furthermore, since $A^{\prime}(t) / A(t)-r(t)=-A(t)^{-1}$, we have

$$
-\int_{t}^{s} A(u)^{-1} d u=\int_{t}^{s} \frac{A^{\prime}(u)}{A(u)} d u-\int_{t}^{s} r(u) d u=\ln A(s)-\ln A(t)-\int_{t}^{s} r(u) d u
$$

and, thus, $\exp \left(-\int_{t}^{s} A(u)^{-1} d u\right)=A(t)^{-1} A(s) \beta(t, s)$. By substituting this into $\tilde{F}(t)$ and integrating by parts, we get

$$
\tilde{F}(t)=A(t)^{-1} \int_{t}^{T} A(s) \beta(t, s) g(s) d s=A(t)^{-1} \int_{t}^{T} \beta(t, s) \int_{t}^{s} g(u) d u d s .
$$

This proves that $V$ defined by (9) solves the HJB equation.

Step II: Admissibility of the optimal control candidates. We then turn to show that the optimal candidate policies indeed are admissible in the sense of Definition 1 . The candidate for the optimal investment strategy is given by

$$
\hat{\theta}_{t}=A(t)\left(\frac{\lambda_{S}(t)}{a \sigma_{S}(t)}-\frac{\rho \sigma_{Y}(t)}{\sigma_{S}(t)}\right) .
$$


Since $A$ converges to zero, Assumptions 1 and 2 ensure the needed integrability of $\hat{\theta}$. The candidate process for optimal consumption is given by

$$
\hat{c}(t, x, y)=A(t)^{-1} x+y+\frac{1}{a} A(t)^{-1} \int_{t}^{T} \beta(t, s) \int_{t}^{s} g(u) d u d s .
$$

Here the dynamics of $X^{(\hat{c}, \hat{\theta})}$ is given by

$$
d X_{t}^{(\hat{c}, \hat{\theta})}=\left[m(t)+\left(r(t)-A(t)^{-1}\right) X_{t}^{(\hat{c}, \hat{\theta})}\right] d t+A(t)\left[a^{-1} \lambda_{S}(t)-\rho \sigma_{Y}(t)\right] d W_{t},
$$

where $m$ is the the above deterministic function, i.e.,

$$
m(t)=A(t) \lambda_{S}(t)\left[a^{-1} \lambda_{S}(t)-\rho \sigma_{Y}(t)\right]-a^{-1} \tilde{F}(t)
$$

The last term in $\hat{c}$ is equal to $a \tilde{F}(t)$ which converges to zero as $t \rightarrow T$ and is therefore integrable. The second term is $Y_{t}$ which is an OU process with nicely behaved mean and variance functions, cf. Assumption 1. The first term, $A(t)^{-1} X_{t}^{(\hat{c}, \hat{\theta})}$, is more complicated because $A(u) \rightarrow 0$ for $u \rightarrow T$. By means of Ito's lemma we find the dynamics

$$
\begin{aligned}
d\left[A(t)^{-1} X_{t}^{(\hat{c}, \hat{\theta})}\right] & =X_{t}^{(\hat{c}, \hat{\theta})}\left[\left(A(t)^{-1}\right)^{2}-r(t) A(t)^{-1}\right] d t+A(t)^{-1} d X_{t}^{(\hat{c}, \hat{\theta})} \\
& =A(t)^{-1} m(t) d t+\left[a^{-1} \lambda_{S}(t)-\rho \sigma_{Y}(t)\right] d W_{t} .
\end{aligned}
$$

From this and Assumptions 1 and 2 it follows that the variance function is finite

$$
\int_{0}^{T}\left(a^{-1} \lambda_{S}(t)-\rho \sigma_{Y}(t)\right)^{2} d t<\infty .
$$

However, the mean function is more complicated. By integrating, we find the mean function to be

$$
\begin{aligned}
\psi(t) & =\mathbb{E}\left[A(t)^{-1} X_{t}^{(\hat{c}, \hat{\theta})}\right] \\
& =A(0)^{-1} X_{0}^{(\hat{c}, \hat{\theta})}+\int_{0}^{t} \lambda_{S}(u)\left[a^{-1} \lambda_{S}(u)-\rho \sigma_{Y}(u)\right] d u-a^{-1} \int_{0}^{t} \frac{\tilde{F}(u)}{A(u)} d u .
\end{aligned}
$$

We wish to show that $\psi$ is a continuous function on $[0, T]$ and that $\psi$ does not blow up at $T$. This follows if we show that the ratio $\tilde{F}(u) / A(u)$ has a finite limit when $u \rightarrow T$ because in that case, $\tilde{F} / A$ is a continuous function on the compact interval $[0, T]$. By the rules of 
Leibnitz and L'Hopital as well as the definition of $\tilde{F}$ we find

$$
\begin{aligned}
\lim _{u \uparrow T} \frac{\tilde{F}(u)}{A(u)} & =\lim _{u \uparrow T} \frac{\tilde{F}(u) A(u)}{A(u)^{2}} \\
& =\lim _{u \uparrow T} \frac{r(u) \tilde{F}(u) A(u)-g(u) A(u)}{2 A(u)[r(u) A(u)-1]} \\
& =\lim _{u \uparrow T} \frac{r(u) \tilde{F}(u)-g(u)}{2[r(u) A(u)-1]}=\frac{1}{2} g(T),
\end{aligned}
$$

which is finite by Assumptions 1 and 2. Therefore, $\tilde{F} / A$ is a continuous function on $[0, T]$ and as a consequence, the mean function $\psi$ is also continuous on $[0, T]$. In conclusion, the needed integrability condition (6) is satisfied.

Lemma 1 stated and proved below shows that $\hat{c}$ and $\hat{\theta}$ satisfy $X_{T}^{(\hat{c}, \hat{\theta})}=0$ and, therefore, we only we need to check the martingality of the two stochastic integrals in (10):

$$
\int_{0}^{t} e^{-\delta u} V_{x} \hat{\theta}_{u} \sigma_{S}(u) d W_{u} \text { and } \int_{0}^{t} e^{-\delta u} V_{y} \sigma_{Y}(u) d Z_{u}
$$

To do so, it suffices to show square integrability of the integrands, i.e., we need

$$
\mathbb{E}\left[\int_{0}^{T}\left(e^{-\delta u} V_{x} \hat{\theta}_{u} \sigma_{S}(u)\right)^{2} d u\right]<\infty \quad \text { and } \quad \mathbb{E}\left[\int_{0}^{T}\left(e^{-\delta u} V_{y} \sigma_{Y}(u)\right)^{2} d u\right]<\infty
$$

By using the explicit form of $V$ and the optimal control candidates $(\hat{c}, \hat{\theta})$ we have

$$
\begin{aligned}
& V_{x} \hat{\theta}_{u} \sigma_{S}(u)=a \exp \left(-a A(u)^{-1} X_{u}^{(\hat{c}, \hat{\theta})}-a Y_{u}-\tilde{F}(u)\right) A(u)\left(\frac{\lambda_{S}(u)}{a}-\rho \sigma_{Y}(u)\right), \\
& V_{y} \sigma_{Y}(u)=a A(u) \exp \left(-a A(u)^{-1} X_{u}^{(\hat{c}, \hat{\theta})}-a Y_{u}-\tilde{F}(u)\right) \sigma_{Y}(u) .
\end{aligned}
$$

Under Assumptions 1 and 2, we can use Cauchy-Schwartz's inequality repeatedly to see that it suffices to show that

$$
\exp \left(A(u)^{-1} X_{u}^{(\hat{c}, \hat{\theta})}\right) \text { and } \exp \left(Y_{u}\right)
$$

have all moments. To argue that this indeed is true, we recall that for a normally distributed random variable $U, U \sim \mathcal{N}\left(\mu, \sigma^{2}\right)$, we have for $p \in \mathbb{R}$ the identity

$$
\mathbb{E}[\exp (p U)]=\exp \left(p \mu+\frac{1}{2} p^{2} \sigma^{2}\right) .
$$

Since both $A(u)^{-1} X_{u}^{(\hat{c}, \hat{\theta})}$ and $Y_{u}$ are normally distributed, this formula shows that it suffices to argue that the mean function and the variance functions are continuous on $[0, T]$ and do not blow up at $T$. Again, because of Assumption 1, this holds for $Y$ 's mean and variance func- 
tion and we just proved that it holds for the mean and variance function for $A(t)^{-1} X_{t}^{(\hat{c}, \hat{\theta})}$ too.

Step III: Verifying that $V$ indeed equals the value function $J$. We first show that we have $V \geq J$ and so we let $(c, \theta) \in \mathcal{A}$ be arbitrary. We can assume that $\mathbb{E}\left[\int_{0}^{T} e^{-a c_{s}} d s\right]<\infty-$ otherwise, there is nothing to prove. Itô's lemma renders the following relation for $t \in[0, T]$ :

$$
\begin{aligned}
e^{-\delta T} V\left(T, X_{T}^{(c, \theta)}, Y_{T}\right) & =e^{-\delta t} V\left(t, X_{t}^{(c, \theta)}, Y_{t}\right)+\int_{t}^{T} e^{-\delta u} \operatorname{drift}_{u}^{(c, \theta)} d u \\
& +\int_{t}^{T} e^{-\delta u} V_{x} \theta_{u} \sigma_{S}(u) d W_{u}+\int_{t}^{T} e^{-\delta u} V_{y} \sigma_{Y}(u)\left(\rho d W_{u}+\sqrt{1-\rho^{2}} d Z_{u}\right)
\end{aligned}
$$

where $e^{-\delta t} \operatorname{drift}_{t}^{(c, \theta)}$ is the $d t$-part of $e^{-\delta t} V\left(t, X_{t}^{(c, \theta)}, Y_{t}\right)$. By the HJB equation we have

$$
\operatorname{drift}_{t}^{(c, \theta)}-e^{-a c t} \leq 0
$$

and, therefore, by taking conditional expectations through and using the martingale property (10) we find

$$
\mathbb{E}_{t}\left[e^{-\delta T} V\left(T, X_{T}^{(c, \theta)}, Y_{T}\right)\right] \leq e^{-\delta t} V\left(t, X_{t}^{(c, \theta)}, Y_{t}\right)+\mathbb{E}_{t}\left[\int_{t}^{T} e^{-\delta u} e^{-a c_{u}} d u\right]
$$

However, by the boundary condition (31) and by the admissibility requirement (7), the above left-hand-side vanishes. All in all, we have the estimate

$$
V\left(t, X_{t}^{(c, \theta)}, Y_{t}\right) \geq \mathbb{E}_{t}\left[\int_{t}^{T}-e^{-\delta(u-t)} e^{-a c_{u}} d u\right]
$$

from which the inequality $V \geq J$ follows. To justify that we have $V=J$, we note that given the admissibility of the optimal control candidates $(\hat{c}, \hat{\theta})$, we can re-do the above calculations but replacing the inequalities with equalities and the claim follows.

Lemma 1 (Ornstein-Uhlenbeck Bridge). Given the assumptions stated in Theorem 1. Let T be a positive constant and let $m$ and $c$ be two continuous finitely valued deterministic functions defined on $[0, T]$. We assume that

$$
\lim _{s \uparrow T} m(s)=0 .
$$

Then there exists a unique stochastic process $X=\left(X_{t}\right)$ solving the affine SDE

$$
d X_{t}=\left(m(t)+\left(r(t)-A(t)^{-1}\right) X_{t}\right) d t+c(t) A(t) d W_{t}, \quad t \in(0, T), \quad X_{0} \in \mathbb{R}
$$

and $X_{t} \rightarrow 0, \mathbb{P}$-a.s., as $t \rightarrow T$. 
Proof. We define the deterministic function $b(t)=r(t)-A(t)^{-1}$ and we note that $b(t) \rightarrow-\infty$ as $t \rightarrow T$. A direct application of Itô's product rule gives us that the stochastic process

$$
X_{s}=e^{\int_{0}^{s} b(u) d u}\left(X_{0}+\int_{0}^{s} e^{-\int_{0}^{t} b(u) d u}\left(m(t) d t+c(t) A(t) d W_{t}\right)\right), \quad s \in[0, T),
$$

satisfies (33). Furthermore, L'Hopital's rule gives us

$$
\lim _{s \uparrow T} \frac{\int_{0}^{s} e^{-\int_{0}^{t} b(u) d u} m(t) d t}{e^{\int_{0}^{s}-b(u) d u}}=\lim _{s \uparrow T} \frac{m(s)}{-b(s)}=0 .
$$

The proof can therefore be concluded by showing

$$
e^{\int_{0}^{s} b(u) d u} M_{s}=e^{\int_{0}^{s} b(u) d u} \int_{0}^{s} e^{-\int_{0}^{t} b(u) d u} c(t) A(t) d W_{t} \rightarrow 0, \quad \mathbb{P} \text {-a.s. }
$$

as $s \uparrow T$. The quadratic variation of $M$ is given by

$$
\langle M\rangle_{s}=\int_{0}^{s} e^{-2 \int_{0}^{t} b(u) d u} \frac{c(t)^{2}}{A(t)^{-2}} d t, \quad s \in[0, T) .
$$

If $\langle M\rangle_{T}<\infty$, we trivially have that $M$ is a continuous martingale on the interval $[0, T]$ and, in particular, $M_{T}$ is a real valued random variable and the claim follows. If $\langle M\rangle_{T}=\infty$, we can use Exercise II.15 in Protter (2004) to see that

$$
0=\lim _{t \uparrow T} \frac{M_{t}}{\langle M\rangle_{t}}, \quad \mathbb{P} \text {-almost surely }
$$

L'Hopital's rule gives us

$$
\lim _{s \uparrow T}\langle M\rangle_{s} e^{\int_{0}^{s} b(u) d u}=\lim _{s \uparrow T} \frac{\int_{0}^{s} e^{-2 \int_{0}^{t} b(u) d u} \frac{c(t)^{2}}{A(t)^{-2}} d t}{e^{-\int_{0}^{s} b(u) d u}}=\lim _{s \uparrow T} \frac{e^{-\int_{0}^{s} b(u) d u} c(s)^{2}}{-b(s) A(s)^{-2}} .
$$

We want to show that this limit is zero and to do so it suffices to show that

$$
\lim _{s \uparrow T} \frac{e^{\int_{0}^{s} A(u)^{-1} d u}}{A(s)^{-2}}=0 .
$$

Since $\frac{\partial}{\partial t} A(t)^{-2}=2 A(t)^{-2}\left(A(t)^{-1}-r(t)\right)$, we have the representation

$$
A(s)^{-2}=A(0)^{-2} e^{2 \int_{0}^{s}\left(A(u)^{-1}-r(u)\right) d u},
$$

from which (35) follows with the use of Assumption 2. 


\section{References}

Aiyagari, S. R. and M. Gertler (1991). Asset Returns with Transactions Costs and Uninsured Individual Risk. Journal of Monetary Economics 27(3), 311-331.

Attanasio, O. P. and M. Paiella (2011). Intertemporal Consumption Choices, Transaction Costs and Limited Participation in Financial Markets: Reconciling Data and Theory. Journal of Applied Econometrics 26(2), 322-343.

Bansal, R. and A. Yaron (2004). Risks for the Long Run: A Potential Resolution of Asset Pricing Puzzles. Journal of Finance 59(4), 1481-1509.

Başak, S. (2000). A Model of Dynamic Equilibrium Asset Pricing with Heterogeneous Beliefs and Extraneous Risk. Journal of Economic Dynamics 85 Control 24(1), 63-95.

Bewley, T. (1986). Stationary Monetary Equilibrium with a Continuum of Independently Fluctuating Consumers. In W. Hildenbrand and A. Mas-Collel (Eds.), Contributions to Mathematical Economics in Honor of Gerald Debreu. North-Holland, Amsterdam.

Bodie, Z., R. C. Merton, and W. F. Samuelson (1992). Labor Supply Flexibility and Portfolio Choice in a Life Cycle Model. Journal of Economic Dynamics 8 Control 16(34), 427-449.

Calvet, L. E. (2001). Incomplete Markets and Volatility. Journal of Economic Theory 98(2), $295-338$.

Campbell, J. Y. (2006). Household Finance. Journal of Finance 61(4), 1553-1604.

Cocco, J. F., F. J. Gomes, and P. J. Maenhout (2005). Consumption and Portfolio Choice over the Life Cycle. Review of Financial Studies 18(2), 491-533.

Cochrane, J. H. (2005). Asset Pricing (Revised ed.). Princeton University Press.

Constantinides, G. M. (2002). Rational Asset Prices. Journal of Finance 57(4), 1567-1591.

Constantinides, G. M., J. B. Donaldson, and R. Mehra (2002). Junior Can't Borrow: A New Perspective on the Equity Premium Puzzle. Quarterly Journal of Economics 117(1), 269-296.

Constantinides, G. M. and D. Duffie (1996). Asset Pricing with Heterogeneous Consumers. Journal of Political Economy 104(2), 219-240.

Cuoco, D. and H. He (1994). Dynamic Equilibrium in Infinite-Dimensional Economies with Incomplete Financial Markets. Working paper, University of Pennsylvania.

David, A. (2008). Heterogeneous Beliefs, Speculation, and the Equity Premium. Journal of Finance 63(1), 41-83. 
Davis, S. J., F. Kubler, and P. Willen (2006). Borrowing Costs and the Demand for Equity over the Life Cycle. Review of Economics and Statistics 88(2), 348-362.

Den Haan, W. J. (2001). The Importance of the Number of Different Agents in a Heterogeneous Asset-pricing Model. Journal of Economic Dynamics $\&$ Control 25(5), 721-746.

Detemple, J., R. Garcia, and M. Rindisbacher (2003). A Monte-Carlo Method for Optimal Portfolios. Journal of Finance 58(1), 401-446.

Duffie, D. and C.-F. Huang (1985). Implementing Arrow-Debreu Equilibria by Continuous Trading of Few Long-lived Securities. Econometrica 53(6), 1337-1356.

Duffie, D. and M. O. Jackson (1990). Optimal Hedging and Equilibrium in a Dynamic Futures Market. Journal of Economic Dynamics $\&$ Control 14(1), 21-33.

Dumas, B., A. Kurshev, and R. Uppal (2009). Equilibrium Portfolio Strategies in the Presence of Sentiment Risk and Excess Volatility. Journal of Finance 64(2), 579-629.

Friedman, M. (1957). A Theory of the Consumption Function. Princeton University Press.

Gallmeyer, M. and B. Hollifield (2008). An Examination of Heterogeneous Beliefs with a Short Sale Constraint. Review of Finance 12(2), 323-364.

Gollier, C. and R. Zeckhauser (2005). Aggregation of Heterogeneous Time Preferences. Journal of Political Economy 113(4), 878-896.

Gourinchas, P.-O. and J. A. Parker (2002). Consumption over the Life Cycle. Econometrica $70(1), 47-89$.

Guo, H. and R. F. Whitelaw (2006). Uncovering the Risk-Return Relation in the Stock Market. Journal of Finance 61(5), 1433-1463.

Heaton, J. and D. Lucas (1996). Evaluating the Effects of Incomplete Markets on Risk Sharing and Asset Pricing. Journal of Political Economy 104(3), 443-487.

Heaton, J. and D. Lucas (2000). Portfolio Choice and Asset Prices: The Importance of Entrepreneurial Risk. Journal of Finance 55(3), 1163-1198.

Henderson, V. (2005). Explicit Solutions to an Optimal Portfolio Choice Problem with Stochastic Income. Journal of Economic Dynamics $\&$ Control 29(7), 1237-1266.

Huggett, M. (1993). The Risk-Free Rate in Heterogeneous-Agent Incomplete-Insurance Economies. Journal of Economic Dynamics \& Control 17(5-6), 953-969.

Jouini, E. and C. Napp (2009). Consensus Consumers and Intertemporal Asset Pricing with Heterogeneous Beliefs. Review of Economic Studies 74(4), 1149-1174.

Judd, K. L. (1985). The Law of Large Numbers with a Continuum of IID Random Variables. Journal of Economic Theory 35(1), 19-25. 
Koijen, R. S. J., T. E. Nijman, and B. J. M. Werker (2010). When Can Life-cycle Investors Benefit from Time-varying Bond Risk Premia? Review of Financial Studies 23(2), $741-780$.

Krueger, D. and H. Lustig (2010). When is Market Incompleteness Irrelevant for the Price of Aggregate Risk (and when is it not)? Journal of Economic Theory 145(1), 1-41.

Krusell, P., T. Mukoyama, and A. A. Smith, Jr. (2010, October). Asset Prices in a Huggett Economy. Working paper.

Krusell, P. and A. A. Smith, Jr. (1997). Income and Wealth Heterogeneity, Portfolio Choice and Equilibrium Asset Returns. Macroeconomic Dynamics 1(2), 387-422.

Levine, D. K. and W. R. Zame (2002). Does Market Incompleteness Matter? Econometrica $70(5), 1805-1839$.

Lucas, D. J. (1994). Asset Pricing with Undiversifiable Income Risk and Short Sales Constraints: Deepening the Equity Premium Puzzle. Journal of Monetary Economics 34(3), $325-341$.

Lynch, A. W. and S. Tan (2011). Labor Income Dynamics at Business-cycle Frequencies: Implications for Portfolio Choice. Forthcoming in Journal of Financial Economics.

Malinvaud, E. (1972). The Allocation of Individual Risks in Large Markets. Journal of Economic Theory 4(2), 312-328.

Malinvaud, E. (1973). Markets for an Exchange Economy with Individual Risks. Econometrica 41(3), 383-410.

Merton, R. C. (1969). Lifetime Portfolio Selection Under Uncertainty: The ContinuousTime Case. Review of Economics and Statistics 51(3), 247-257.

Merton, R. C. (1971). Optimum Consumption and Portfolio Rules in a Continuous-Time Model. Journal of Economic Theory 3(4), 373-413.

Munk, C. and C. Sørensen (2010). Dynamic Asset Allocation with Stochastic Income and Interest Rates. Journal of Financial Economics 96(3), 433-462.

Nielsen, L. T. and M. Vassalou (2006). The Instantaneous Capital Market Line. Economic Theory 28(3), 651-664.

Protter, P. (2004). Stochastic Integration and Differential Equations (Second ed.). Springer.

Santos, T. and P. Veronesi (2006). Labor Income and Predictable Stock Returns. Review of Financial Studies 19(1), 1-44.

Storesletten, K., C. I. Telmer, and A. Yaron (2007). Asset Pricing with Idiosyncratic Risk and Overlapping Generations. Review of Economic Dynamics 10(4), 519-548. 
Svensson, L. E. O. and I. M. Werner (1993). Nontraded Assets in Incomplete Markets. European Economic Review 37(5), 1149-1168.

Szpiro, G. G. (1986). Measuring Risk Aversion: An Alternative Approach. Review of Economic Studies 68(1), 156-159.

Telmer, C. I. (1993). Asset-Pricing Puzzles and Incomplete Markets. Journal of Finance 48(5), 1803-1832.

Teplá, L. (2000). Optimal Hedging and Valuation of Nontraded Assets. European Finance Review 4(3), 231-251.

Uhlig, H. (1996). A Law of Large Numbers for Large Economies. Economic Theory 8(1), $41-50$.

Viceira, L. M. (2001). Optimal Portfolio Choice for Long-Horizon Investors with Nontradable Labor Income. Journal of Finance 56(2), 433-470.

Wang, N. (2003). Caballero Meets Bewley: The Permanent-Income Hypothesis in General Equilibrium. American Economic Review 93(3), 927-936.

Wang, N. (2004). Precautionary Saving and Partially Observed Income. Journal of Monetary Economics 51(8), 1645-1681.

Wang, N. (2006). Generalizing the Permanent-Income Hypothesis: Revisiting Friedman's Conjecture on Consumption. Journal of Monetary Economics 53(4), 737-752.

Wang, N. (2009). Optimal Consumption and Asset Allocation with Unknown Income Growth. Journal of Monetary Economics 56(4), 524-534.

Weil, P. (1989). The Equity Premium Puzzle and the Risk-free Rate Puzzle. Journal of Monetary Economics 24(3), 401-421.

Wilson, R. (1968). The Theory of Syndicates. Econometrica 36(1), 119-132.

Xiong, W. and H. Yan (2010). Heterogeneous Expectations and Bond Markets. Review of Financial Studies 23(4), 1433-1466. 